Article

\title{
Synthesis, In Silico Prediction and In Vitro Evaluation of Antitumor Activities of Novel Pyrido[2,3-d]pyrimidine, Xanthine and Lumazine Derivatives
}

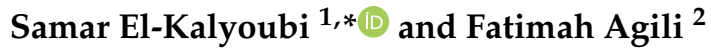 \\ 1 Department of Pharmaceutical Organic Chemistry, Faculty of Pharmacy (Girls), Al-Azhar University, \\ Nasr City, Cairo 11651, Egypt \\ 2 Chemistry Department, Faculty of Science (Female Section), Jazan University, Jazan 82621, Saudi Arabia; \\ fatmah2000@gmail.com \\ * Correspondence: s.elkalyoubi@hotmail.com; Tel.: +20-111-995-2620
}

Academic Editors: György Keglevich and Pascale Moreau

Received: 17 September 2020; Accepted: 30 October 2020; Published: 9 November 2020

\begin{abstract}
Ethyl 5-arylpyridopyrimidine-6-carboxylates 3a-d were prepared as a one pot three component reaction via the condensation of different aromatic aldehydes and ethyl acetoacetate with 6-amino-1-benzyluracil 1a under reflux condition in ethanol. Additionally, condensation of ethyl 2-(2-hydroxybenzylidene) acetoacetate with 6-amino-1-benzyluracil in DMF afforded 6-acetylpyridopyrimidine-7-one $3 \mathbf{e}$; a facile, operationally, simple and efficient one-pot synthesis of 8-arylxanthines $\mathbf{6 a}-\mathbf{f}$ is reported by refluxing 5,6-diaminouracil 4 with aromatic aldehydes in DMF. Moreover, 6-aryllumazines $7 \mathbf{a}-\mathbf{d}$ was obtained via the reaction of 5,6-diaminouracil with the appropriate aromatic aldehydes in triethyl orthoformate under reflux condition. The synthesized compounds were characterized by spectral $\left({ }^{1} \mathrm{H}-\mathrm{NMR},{ }^{13} \mathrm{C}-\mathrm{NMR}\right.$, IR and mass spectra) and elemental analyses. The newly synthesized compounds were screened for their anticancer activity against lung cancer A549 cell line. Furthermore, a molecular-docking study was employed to determine the possible mode of action of the synthesized compounds against a group of proteins highly implicated in cancer progression, especially lung cancer. Docking results showed that compounds $\mathbf{3 b}, \mathbf{6 c}$, $6 \mathrm{~d}, 6 \mathrm{e}, 7 \mathrm{c}$ and $7 \mathrm{~d}$ were the best potential docked compounds against most of the tested proteins, especially CDK2, Jak2, and DHFR proteins. These results are in agreement with cytotoxicity results, which shed a light on the promising activity of these novel six heterocyclic derivatives for further investigation as potential chemotherapeutics.
\end{abstract}

Keywords: 6-amino-1-benzyluracil; 6-Amino-1-(2-chlorobenzyl)uracil; 5,6-diamino-1-benzyl uracil; ethyl 5-aryl-7-methylpyridopyrimidine-6-carboxylate; 8-aryl-3-(2-chlorobenzyl) xanthines and 6-aryl-1-(2-chlorobenzyl)lumazines; cancer; molecular docking

\section{Introduction}

Pulmonary cancer among the two sexes has been the leading cause of cancer lung death for many years and worldwide statistics on incidence and mortality vary widely [1-3]. The world's high mortality rate from malignant tumors is attributed to the uncontrolled growth of cells in lung tissues and to the high metastatic ability of lung cancer [4,5]. Tobacco consumption is a significant lung cancer risk factor. Genetic susceptibility, diet, alcohol consumption, industrial exposures and air pollution are other factors [6]. Pulmonary cancer is primarily caused by the abnormal growth of either small or non-small lung cancer cells [7-10]. In 2015, 80-85\% of cases of lung cancer worldwide were attributed to non-small cell lung cancer (NSCLC) [11]. The main treatment for early lung cancer 
is surgical resection. Radiation is one of the most common methods of treating tumors. In case of advanced diseases (stage IIIB and stage IV), radiotherapy, targeted therapy and immunotherapy are applied [1]. Chemotherapy is one of the most common treatment methods for tumors [12,13]. However, current chemotherapeutic therapies appear to have several limitations, including a lack of tumor cell selectivity [14,15], significant toxic side effects on healthy tissue [16] and drug resistance [17] leading to unsatisfactory therapeutic effects.

The first form of antimetabolites introduced in the clinic 60 years ago was antifolate drugs [18,19]. Methotrexate (MTX) (B) is a folic acid (A) antagonist required for DNA synthesis, and has a therapeutic effect on many cancer cell types that over-express folate receptors on the surfaces of many of these cells (Figure 1) [20]. MTX inhibits the cellular folate metabolism by dihydrofolate reductase (DHFR) in a competitive way. By inhibiting the enzyme target DHFR [21], MTX suppresses purine and pyrimidine synthesis. It acts on S-phase and prevents DNA synthesis [22,23]. The chemotherapeutic MTX is widely used in human malignancies, including acute lymphoblastic leukemia, malignant lymphoma, lung cancer, breast cancer, osteosarcoma and head and neck cancer [24-27]. MTX has been also widely used for the treatment of rheumatoid arthritis (RA) through the release of adenosine-mediated suppression inflammation [28]. Clinical data supports the adenosine-mediated anti-inflammatory effect of MTX [29].

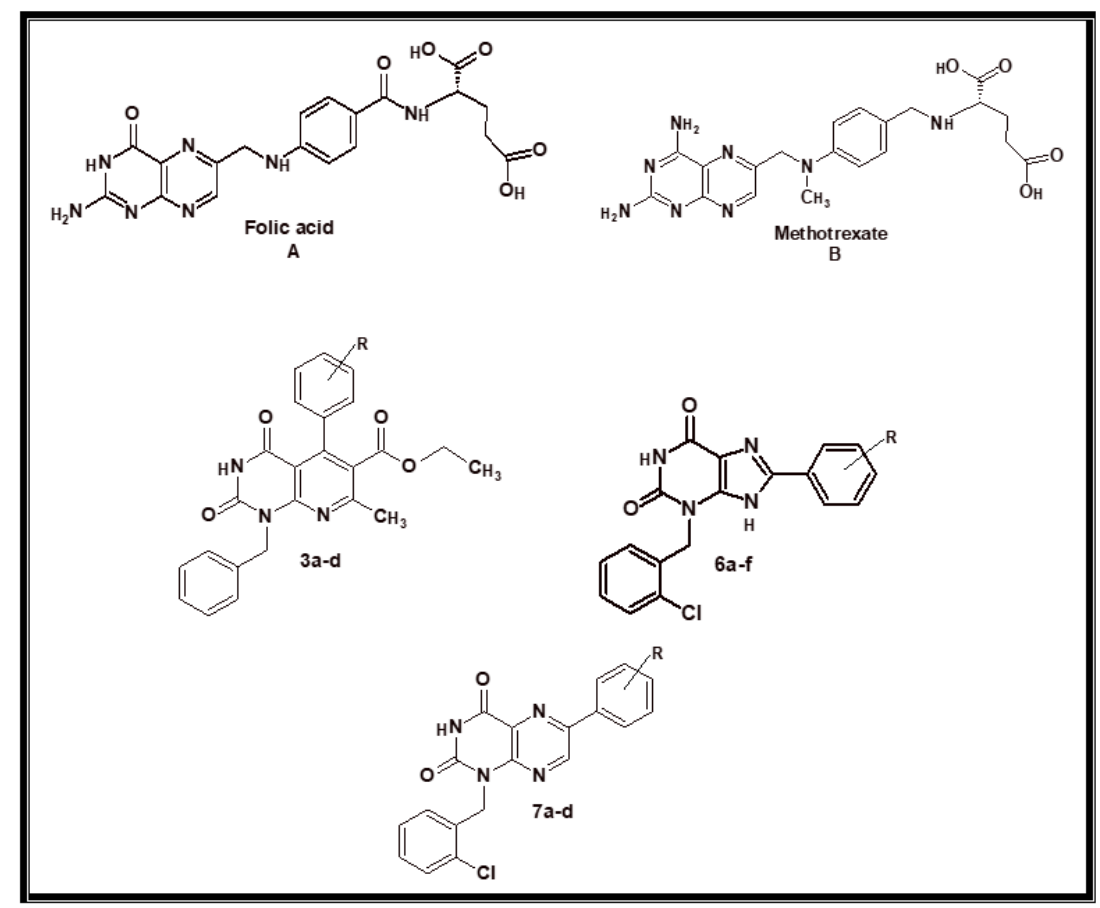

Figure 1. Structure of folic acid, methotrexate and the proposed derivatives.

Chronic neurotoxicity can be caused by MTX [30]. It can also cause alveolitis and lung fibroblasting because of its pulmonary toxicity side effect [31,32]. Hoping to overcome the above mentioned chemotherapeutic limitations, a new series of pyridopyrimidines, xanthines and lumazines have been synthesized (Figure 1). All the newly synthesized compounds herein underwent a molecular-docking study and were screened for their anticancer activity against the A549 cell line.

\section{Results}

\subsection{Chemistry}

In this research, our strategy was directed towards developing new fused uracil derivatives of potential anticancer activity [33,34]. The desired starting materials, including 6-amino-1-benzyluracil 
1a [35] and 5,6-diamino-1-(2-chlorobenzyl)uracil 4 [36] were synthesized according to the literature by conventional methods.

Ethyl 5-aryl-2-thiopyridopyrimidine-6-carboxylates were prepared by heating under reflux of 6-amino-1-methyl-2-thiouracil with ethyl 3-aryl-2-cyanoacrylate in absolute ethanol in the presence of triethylamine (TEA) [37]. Ethyl 5-aryl-2-pyridopyrimidine-6-carboxylates 3a-d were prepared in higher yields (60-84\%) by refluxing compound 1a with different aromatic aldehydes, ethyl acetoacetate in abs. ethanol containing TEA as a basic medium for $12 \mathrm{~h}$ as illustrated in (Scheme 1). The derivatives were crystallized from DMF/EtOH. Analogously, the treatment of compound 1a with ethyl 2-(2-hydroxybenzylidene)acetoacetate in DMF in the presence of TEA for $4 \mathrm{~h}$ gave 6-acetyl-5-arylpyridopyrimidine 7-one $3 \mathbf{e}$ (Scheme 1). The most interesting observation from comparing the ${ }^{1} \mathrm{H}-\mathrm{NMR}$ spectra of compounds $3 \mathbf{a}-\mathbf{d}$ and compound $3 \mathbf{e}$ was the disappearance of both signals of $\mathrm{CH}-5$ at $\delta 5.81 \mathrm{ppm}$ and $\mathrm{NH}_{2}$ (6) at $\delta 5.97 \mathrm{ppm}$ of the start $1 \mathrm{a}$ and the appearance of ethyl protons at position 6 in pyridopyrimidines $3 \mathbf{a}-\mathbf{d}$ as triplet at $\delta 1.09-0.96 \mathrm{ppm}$ for $\mathrm{CH}_{3}$ and quartet at $\delta$ 4.18-3.94 for $\mathrm{CH}_{2}$. A singlet signal occurred at $\delta 2.90 \mathrm{ppm}$ for $\mathrm{CH}_{3}$ protons of acetyl group at position 6 in pyridopyrimidine $3 \mathbf{e}$ and a singlet signal at $\delta 7.88 \mathrm{ppm}$ for $\mathrm{NH}(8)$. On the other hand, the IR spectra of these pyridopyrimidines, which displayed the stretching band of the two $\mathrm{C}=\mathrm{O}$ groups (Amide I), was red-shifted within the range $v 1745-1723,1684-1697 \mathrm{~cm}^{-1}$. We noted the strong asymmetric and symmetric $\mathrm{NO}_{2}$ stretching bands of the nitro group at $v 1551$ and $1374 \mathrm{~cm}^{-1}$, respectively, in compound 3d. ${ }^{13} \mathrm{C}-\mathrm{NMR}$ spectra proves without doubt the formation of compounds $3 \mathbf{a}-\mathbf{d}$ through the appearance of the upfield signals of ethyl group of ester at $\delta 14.02-13.43 \mathrm{ppm}$ for $\mathrm{CH}_{3}$ and at $\delta 61.81-59.55 \mathrm{ppm}$ for $\mathrm{CH}_{2}$, in addition to the presence of a signal at $\delta 18.13-17.52 \mathrm{ppm}$ for $\mathrm{CH}_{3}(7)$. While in comparison with the ${ }^{13} \mathrm{C}$-NMR spectra of compound $3 \mathbf{e}$, it was observed that the signal of $\mathrm{CH}_{3}$ of acetyl group was $\delta$ $17.53 \mathrm{ppm}$.

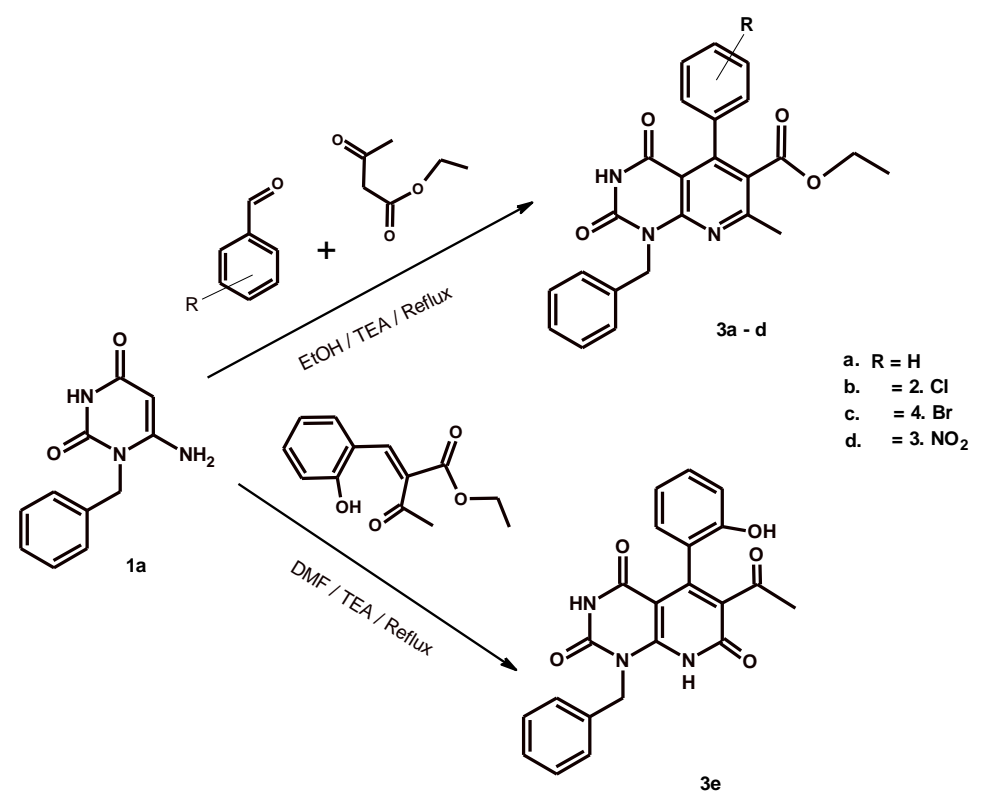

Scheme 1. Formation of pyridopyrimidines from 6-amino-1-benzyluracil.

The mechanism outlined in Scheme 2 seems to be the most plausible pathway through the Michael addition reaction via the formation of non-isolated Michael adduct intermediate that underwent intramolecular cyclization in protic solvent affording the corresponding $\mathbf{3 a - d}$. On the other hand, Scheme 3 illustrates the mechanistic pathway for the formations of compound $3 \mathbf{e}$ via the formation of Michael adduct intermediate followed by cyclocondensation in aprotic solvent. 


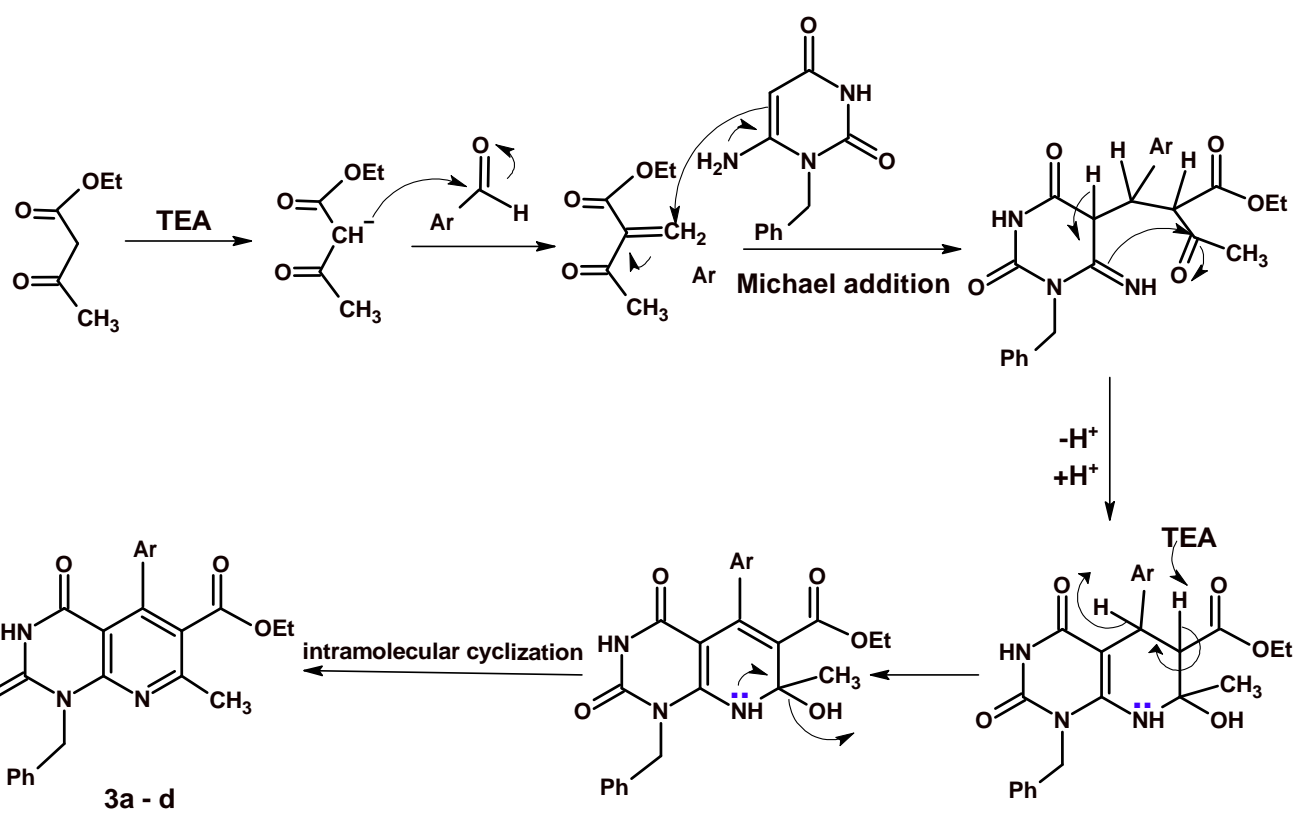

Scheme 2. Mechanistic pathway for the formation of ethyl pyridipyrimidine-6-carboxylate 3a-d.

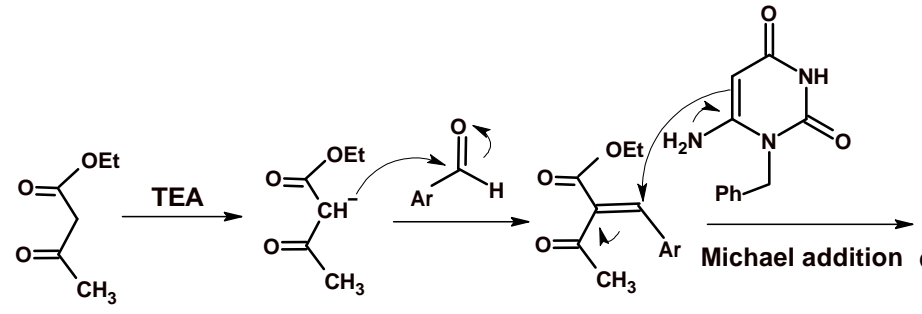

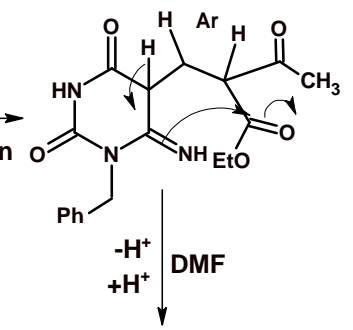<smiles>CC(=O)c1c(O)nc2c(c1Br)c(=O)[nH]c(=O)n2Cc1ccccc1</smiles><smiles></smiles>

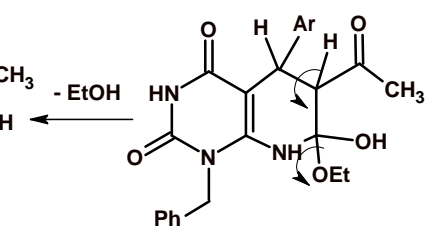<smiles>CC(=O)c1c(Br)c2c(=O)[nH]c(=O)n(Cc3ccccc3)c2[nH]c1=O</smiles>

Scheme 3. Mechanistic pathway for the formation of 6-acetyl pyridipyrimidine-2,4,7-trione 3e.

8-Aryl xanthines have been reported [36,38]. Herein, condensation of 5,6-diaminouracil 4 with different aromatic aldehydes in DMF under reflux for two to three hours afforded 8-aryl xanthine derivatives 6a-f (Scheme 4). The reaction proceeded through the nucleophilic attack of the amino group at position 5 of uracil $\mathbf{1 b}$ to the electrophilic center of the carbonyl group of the aromatic aldehydes followed by dehydration to form the intermediate Schiff base which undergoes intramolecular cyclization to afford $\mathbf{6 a}-\mathbf{f}$ in good to excellent yield. The intrinsic significance of the IR spectra is that the presence of the stretching band of the $2 \mathrm{C}=\mathrm{O}$ groups (Amide I) within the range $v 1684,1643 \mathrm{~cm}^{-1}$. 
${ }^{1} \mathrm{H}-\mathrm{NMR}$ spectra prove the suggested xanthines $\mathbf{6 a}-\mathbf{f}$ by the presence of both characteristic singlet signals for $\mathrm{NH}(9)$ at $\delta 14.46-13.23 \mathrm{ppm}$ and $\mathrm{NH}(1)$ at $\delta 1139-10.94 \mathrm{ppm}$. The phenyl group protons appeared at $\delta 8.60-6.81 \mathrm{ppm}$. The clearance of the spectra from the signals at $\delta 6.78 \mathrm{ppm}$ and at $\delta$ $5.79 \mathrm{ppm}$, characteristic for $2 \mathrm{NH}_{2}$ protons of uracil 4 at position 5 and 6, respectively, supported the previous observation. Also, the ${ }^{13} \mathrm{C}-\mathrm{NMR}$ spectra and Mass spectra supported the previous expectation. With these results in hand, xanthines $\mathbf{6 a}-\mathbf{f}$ became confirmed without a doubt.<smiles>Nc1cc(=O)[nH]c(=O)n1Cc1ccccc1Cl</smiles>

$1 \mathrm{~b}$

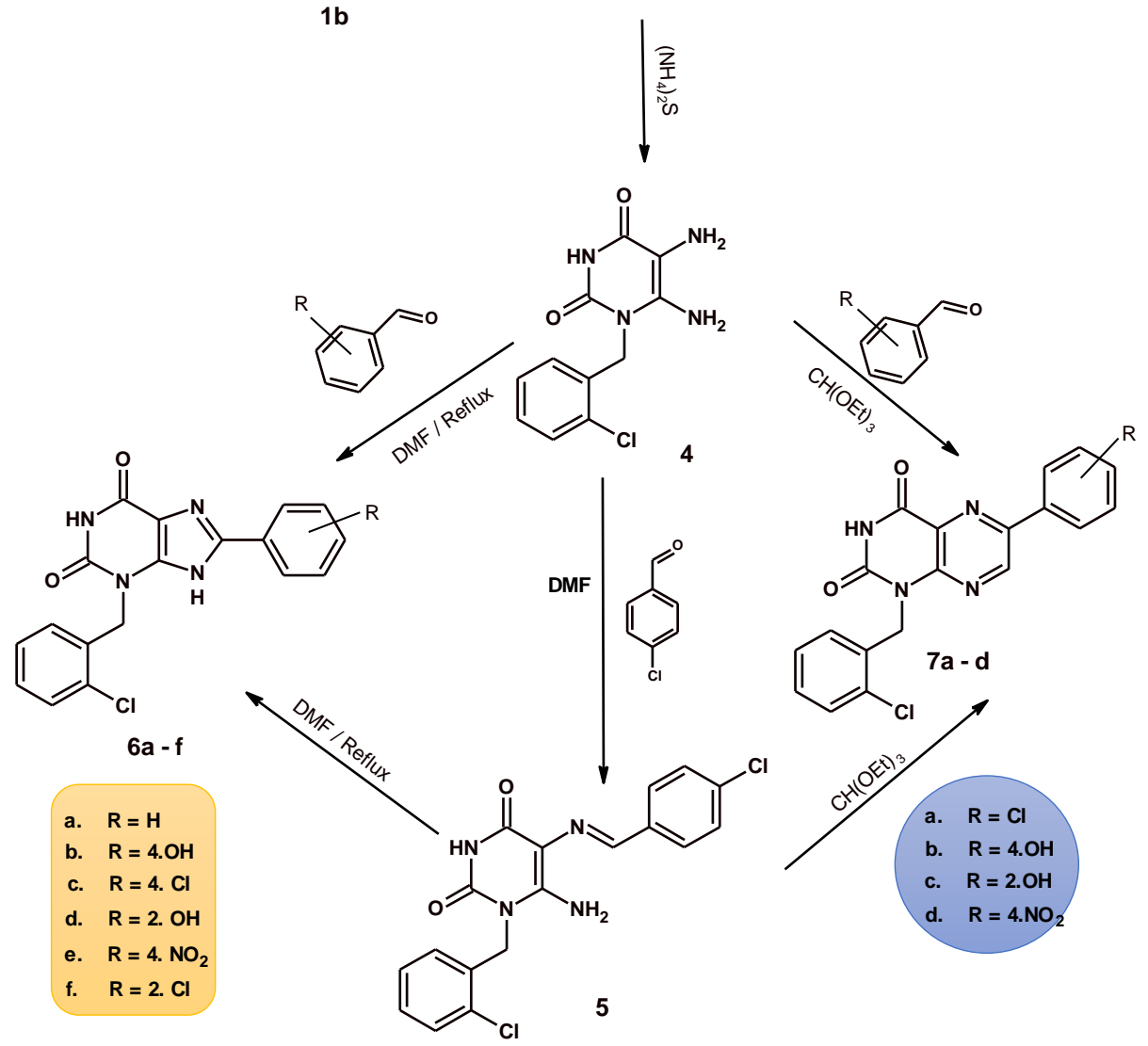

Scheme 4. Formation of xanthines and lumazines from 6-aminouracils.

Moreover, heating of 5,6-diaminouracil 4 with various aromatic aldehydes in triethyl orthoformate under reflux for 7-8 $\mathbf{h}$ afforded 6-aryllumazines $7 \mathbf{a}-\mathbf{d}$ (Scheme 4). Structures $7 \mathbf{a}-\mathbf{d}$ were inferred from their spectral data, and elemental analyses. ${ }^{1} \mathrm{H}-\mathrm{NMR}$ data for compound $\mathbf{7 a - d}$ showed a singlet signal at $\delta 12.01-11.29 \mathrm{ppm}$ for NH-3 and a singlet signal at $\delta 8.71-8.70 \mathrm{ppm}$ for $\mathrm{CH}-7$ in lumazine ring. The IR spectrum revealed bands at the range of $v 3141-3127 \mathrm{~cm}^{-1}$ characteristic for $\mathrm{NH}$. Compound $\mathbf{7 b}$ showed a characteristic band for $\mathrm{OH}$ at $v 3480 \mathrm{~cm}^{-1}$. The mechanistic pathway is illustrated via the reaction of the intermediate Schiff base 5 with triethyl orthoformate followed by intramolecular cycloaddition accompanied by elimination of $\mathrm{EtOH}$ (Scheme 5). The plausible mechanism was proved by isolating the intermediate Schiff base 5 with its interesting spectral data. Otherwise, compound 5 was refluxed with DMF afforded xanthine $\mathbf{6 c}$, while refluxing with triethyl orthoformate afforded the lumazine $7 \mathbf{b}$. 


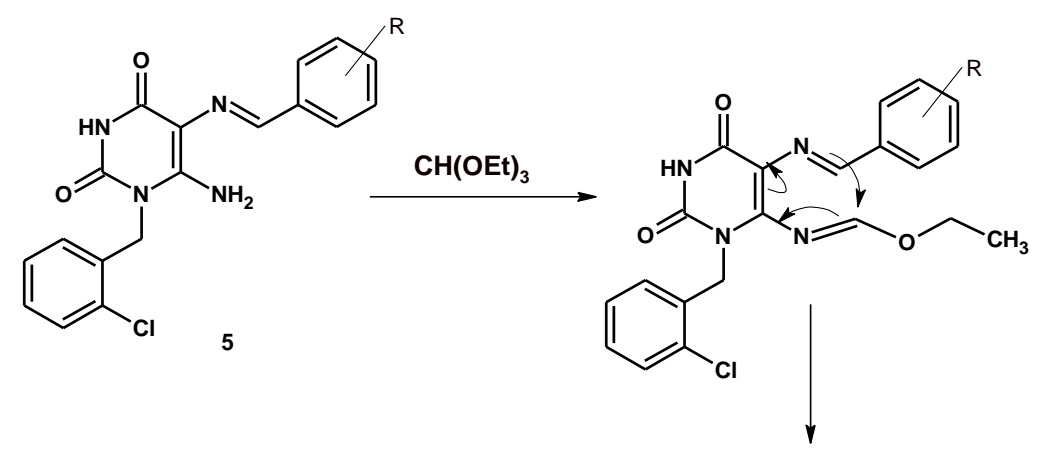

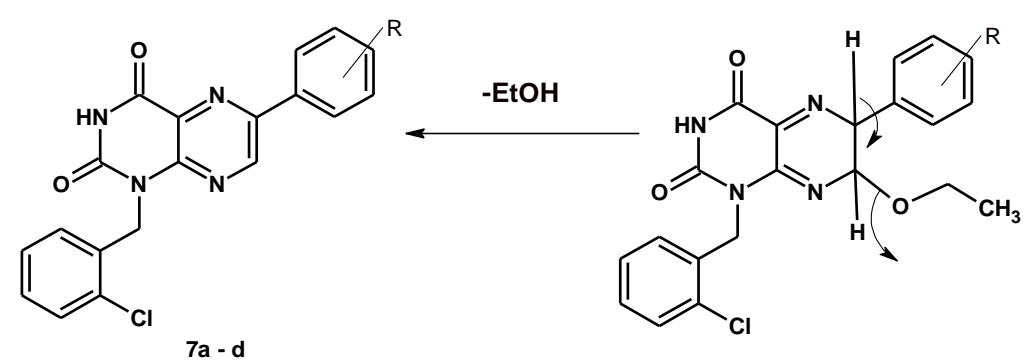

Scheme 5. Mechanistic pathway to the formation of 6-aryllumazines.

\subsection{Biological Activity}

Anticancer Evaluation

During initial cytotoxic screening for the tested compounds, we tested the activity of the compounds against two cell lines, which were lung (A549 cell line) and breast cancer (MCF7). The cytotoxic activity against lung cancer was potentially more promising than breast cancer. Therefore, we have selected the lung cancer cell line.

All the newly synthesized compounds were screened for their cytotoxic effects on the human cell lung adenocarcinoma A549 cell line. The obtained $\mathrm{IC}_{50}$ values of the synthesized compounds were compared to well-known reference anticancer drug methotrexate under the same conditions using a colorimetric viability assay. $\mathrm{IC}_{50}$ values were determined from plots of a dose response curve of the concentration of test compounds required to kill $50 \%$ of cell population. The in vitro growth inhibition results showed that all the tested compounds have an inhibitory effect on the tumor cell line A549 in a concentration dependent manner. All the results are represented in Table 1 and Figure 2.

Table 1. In vitro inhibitory activity of tested compounds against the human Lung carcinoma cell line (A549) expressed as IC50 values $\mu \mathrm{M} \pm$ standard deviation.

\begin{tabular}{cc}
\hline Tested Compounds & IC $_{\mathbf{5 0}}$ Values $(\boldsymbol{\mu M})$ \\
\hline $\mathbf{3 a}$ & $237 \pm 6.3$ \\
$\mathbf{3 b}$ & $10.3 \pm 0.2$ \\
$\mathbf{3 c}$ & $349 \pm 7.8$ \\
$\mathbf{3 d}$ & $59.5 \pm 2.5$ \\
$\mathbf{3 e}$ & $246 \pm 7.1$ \\
$\mathbf{5}$ & $62.0 \pm 2.4$ \\
$\mathbf{6 a}$ & $54.0 \pm 1.8$ \\
$\mathbf{6 b}$ & $58.5 \pm 1.7$ \\
$\mathbf{6 c}$ & $27.0 \pm 1.1$ \\
$\mathbf{6 d}$ & $23.1 \pm 0.6$ \\
$\mathbf{6 e}$ & $26.3 \pm 1.3$ \\
$\mathbf{6 f}$ & $141 \pm 3.9$ \\
$\mathbf{7 a}$ & $86.1 \pm 2.8$ \\
$\mathbf{7 b}$ & $84.8 \pm 3.4$ \\
$\mathbf{7 c}$ & $24.9 \pm 1.2$ \\
$\mathbf{7 d}$ & $12.2 \pm 0.3$ \\
Methotrexate & $36.3 \pm 3.9$
\end{tabular}



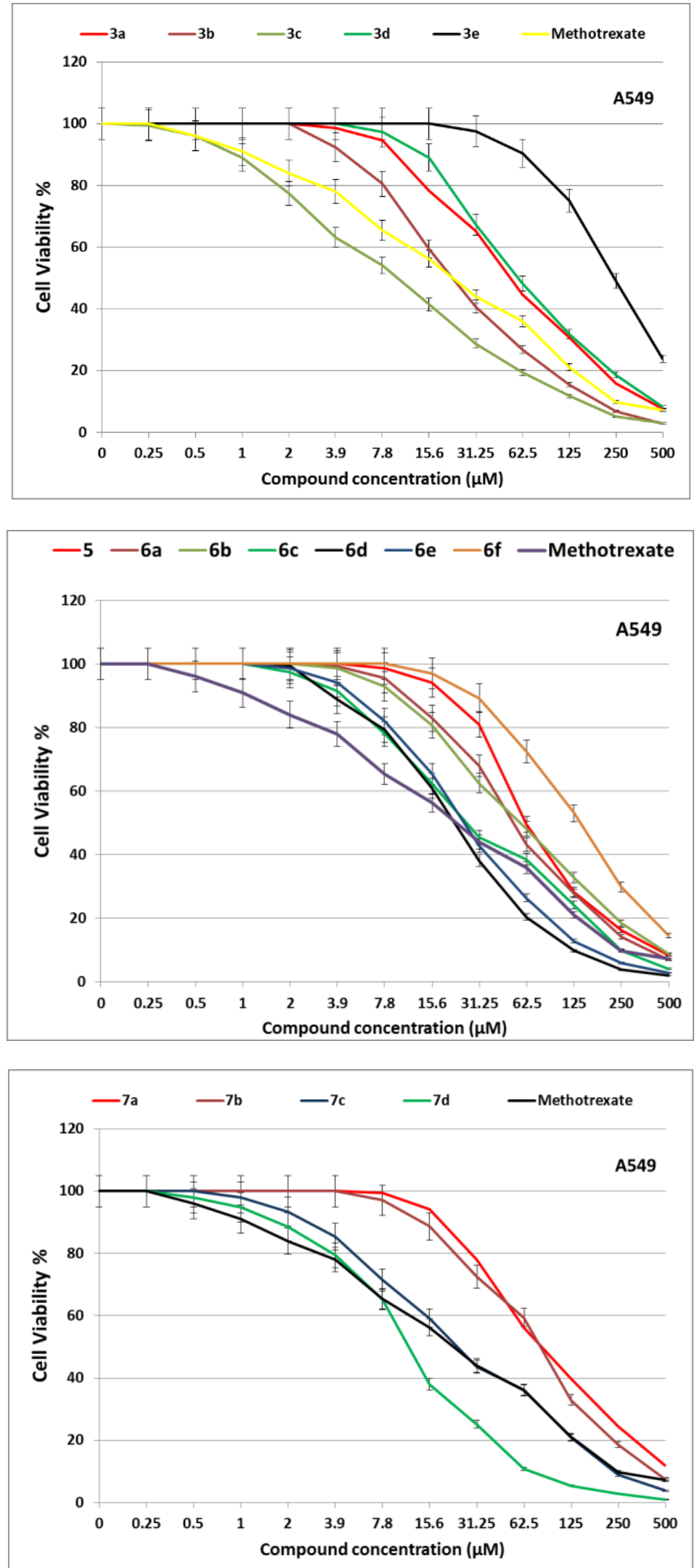

Figure 2. Growth inhibition curves showing the human Lung adenocarcinoma (A549) cell line treated with the tested compounds at different concentrations compared to the reference drug methotrexate. 
It was noticed that compounds $\mathbf{3 b}$ and $\mathbf{7 d}$ exhibited the highest inhibitory activity against the Lung carcinoma cell line (A549) in comparison with the reference drug methotrexate with IC $_{50}$ values of 10.3 and $12.2 \mu \mathrm{M}$, respectively. Also, compounds $\mathbf{6 c}-\mathbf{6 e}$ and $7 \mathrm{c}$ showed potent antitumor activity against the Lung carcinoma cell line (A549) with $\mathrm{IC}_{50}$ values of $23.1,26.3,27$ and $24.9 \mu \mathrm{M}$, respectively. The other compounds have less antitumor activity (Table 1 and Figure 2).

\subsection{Computer-Aided Docking}

To take one step further to determine the mode of action of the tested compounds, a molecular docking study was employed to determine the binding modes against series of proteins such as CDK2, BCL2, Jak2, P53 binding site in MDM2, and DHFR that are implicated significantly in cancer disease (Figures S1-S5). These targets were selected based on their potential roles in apoptosis regulation and limiting lung cancer progression, therefore, targeting these macromolecules provides potential benefits in lung cancer therapy. The co-crystal ligands were re-docked to assure the validity of the docking parameters and methods used to represent the position and orientation of the ligand detected in the crystal structure. The difference of RMSD value between co-crystal ligands to the original co-crystal ligand was $<2 \AA$ which approved the accuracy of the docking protocols and parameters [39].

The docking results depicted that all tested compounds showed poor binding affinity with BCL-2 protein, as the binding free energy of all tested compounds was higher than the binding free energy of co-crystalized ligands $\left(\Delta \mathrm{G}_{\mathrm{b}}=-10.6 \mathrm{kcal} / \mathrm{mol}\right)$. In the same line, all tested compounds had low binding affinity with MDM2-P53 protein, except compounds $\mathbf{6 d}$ and $\mathbf{7 c}$, as they had lower binding free energy $(-8.3 \mathrm{kcal} / \mathrm{mol}$, and $-8.4 \mathrm{kcal} / \mathrm{mol}$, respectively) compared to [(3R,5R,6S)-5-(3-chlorophenyl)-6-(4-chlorophenyl)-1-(cyclopropylmethyl)-2-oxopiperidin-3-yl] acetic acid which is abbreviated as $13 \mathrm{Q}$ the co-crystalized ligand $\left(\Delta \mathrm{G}_{\mathrm{b}}=-8.2 \mathrm{kcal} / \mathrm{mol}\right)$ (Table 2) (Supplementary Figures S2 and S4). Based on these results, the mode of action of the tested compound might not be related to both BCL-2 and MDM2-P53 proteins. All tested compounds showed high binding affinity against $C D K 2$ proteins, as all tested compounds showed low binding free energies compared to both reference drug $\left(\Delta \mathrm{G}_{\mathrm{b}}=-8.3 \mathrm{kcal} / \mathrm{mol}\right)$ and methotrexate $\left(\Delta \mathrm{G}_{\mathrm{b}}=-7.6 \mathrm{kcal} / \mathrm{mol}\right)$, which implies a potential inhibitory effect of the tested compounds against the cyclin dependent kinase enzyme. 
Table 2. Binding Free energy for the tested compounds against docked proteins (CDK2, BCL2, Jak2, MDM2-P53, and DHFR) binding pockets in comparison to the co-crystallized ligands and methotrexate.

\begin{tabular}{|c|c|c|c|c|c|}
\hline \multirow{3}{*}{ Comp. No } & CDK2 & BCL2 & Jak2 & MDM2-P53 & DHFR \\
\hline & PDB: 1DI8 & PDB: $2 \mathrm{O} 2 \mathrm{~F}$ & PDB: 5AEP & PDB: 2LZG & PDB: 4DFR \\
\hline & $\Delta \mathrm{G}_{\mathrm{b}}{ }^{\mathrm{a}}$ & $\Delta \mathrm{G}_{\mathrm{b}}^{\mathrm{a}}$ & $\Delta \mathrm{G}_{\mathrm{b}}{ }^{\mathrm{a}}$ & $\Delta \mathrm{G}_{\mathrm{b}}^{\mathrm{a}}$ & $\Delta \mathrm{G}_{\mathrm{b}}{ }^{\mathrm{a}}$ \\
\hline $3 a$ & -8.5 & -7.8 & -7.6 & -7.6 & -8.7 \\
\hline $3 b$ & -8.7 & -7.7 & -7.7 & -7.0 & -8.8 \\
\hline $3 c$ & -8.9 & -7.8 & -7.7 & -7.0 & -8.6 \\
\hline $3 d$ & -9.3 & -7.1 & -8.1 & -7.0 & -8.5 \\
\hline $3 e$ & -8.6 & -7.7 & -8.7 & -7.7 & -8.7 \\
\hline 5 & -8.8 & -8.5 & -9.5 & -7.8 & -8.2 \\
\hline $6 a$ & -9.7 & -8.5 & -9.3 & -7.8 & -8.5 \\
\hline $6 b$ & -9.6 & -8.5 & -9.6 & -7.9 & -8.6 \\
\hline $6 c$ & -9.3 & -8.9 & -9.6 & -7.9 & -8.8 \\
\hline $6 \mathrm{~d}$ & -10.1 & -8.5 & -9.4 & -8.4 & -8.6 \\
\hline $6 e$ & -9.8 & -8.9 & -9.7 & -7.8 & -9.1 \\
\hline $6 f$ & -9.0 & -8.9 & -9.6 & -8.0 & -8.6 \\
\hline $7 a$ & -9.7 & -8.5 & -9.8 & -8.1 & -8.8 \\
\hline $7 \mathbf{b}$ & -9.2 & -8.5 & -9.7 & -7.8 & -8.6 \\
\hline $7 \mathrm{c}$ & -9.2 & -8.5 & -9.7 & -8.3 & -9.2 \\
\hline $7 d$ & -9.5 & -8.8 & -9.8 & -7.6 & -8.7 \\
\hline Methotrexate & -7.6 & -8.0 & -9.0 & -6.9 & -7.8 \\
\hline Reference ligand & -8.3 & -10.6 & -9.1 & -8.2 & -7.8 \\
\hline
\end{tabular}

${ }^{\mathrm{a}} \Delta \mathrm{G}_{\mathrm{b}}$ : binding Free energy $(\mathrm{kcal} / \mathrm{mol})$.

Based on the molecular docking study, we tested the proposed activity of the tested compounds against DHFR protein, which is the main target for MTX. Compounds $\mathbf{3 b}, \mathbf{6 c}, \mathbf{6 d}, \mathbf{6 e}, \mathbf{7 c}$ and $\mathbf{7 d}$ were the best docked compounds against CDK2, Jak2, and DHFR proteins, which are displayed by low binding free energy (Table 2), hydrogen bond formation (Tables 3-5), and hydrophobic interaction (Figures 3 and 4, Figures S6-S8) compared to reference ligand and methotrexate. These results are in agreement with the cytotoxicity assay as compounds $3 \mathbf{b}, \mathbf{6 c}, \mathbf{6 d}, \mathbf{6 e}, \mathbf{7} \mathbf{c}$ and $\mathbf{7 d}$ showed lower $\mathrm{IC}_{50 \mathrm{~s}}(10.3 \pm 0.2$, $27.0 \pm 1.1,23.1 \pm 0.6,26.3 \pm 1.3,24.9 \pm 1.2,12.2 \pm 0.3 \mu \mathrm{M}$, respectively) compared to methotrexate as a reference drug $(36.3 \pm 3.9 \mu \mathrm{M})$.

Table 3. Hydrogen bond and hydrophobic interaction for the most promising tested compounds into CDK2 binding pocket in comparison to the co-crystallized ligand.

\begin{tabular}{ccccc}
\hline \multirow{2}{*}{ Comp. No } & \multicolumn{3}{c}{ Hydrogen Bonding } & \multicolumn{2}{c}{ Types of Interactions } \\
\cline { 2 - 5 } & No & Length $\mathbf{A}$ & AA $^{\text {a }}$ & Hydrophobic $^{\text {a }}$ \\
\hline 3b & 1 & 2.040 & ASP86 & ILE10, VAL18, VAL64, LEU298, LEU134, LEU83, LEU133, PHE80, PHE82 \\
6c & 1 & 2.129 & LEU83 & ILE10, VAL18, VAL64, LEU134, LEU83, LEU148, PHE80, PHE82 \\
& & 3.137 & LEU83 & \\
6d & 2 & 3.245 & LYS33 & ILE10, VAL18, VAL64, LEU134, LEU83, LEU148, PHE80, PHE82 \\
6e & 1 & 2.329 & ASP145 & ILE10, VAL18, VAL64, LEU134, LEU83, PHE80, PHE82 \\
7c & 2 & 3.350 & LEU83 & ILE10, VAL18, VAL64, LEU134, LEU83, LEU148, PHE80, PHE82 \\
7d & 1 & 2.499 & GLU12 & ILE10, VAL18, VAL64, LEU134, LEU83, LEU298, PHE80, PHE82 \\
RL b & 2 & 2.831 & LYS33 & ILE10, VAL18, VAL64, LEU148, LEU83, LEU134, PHE82 \\
\end{tabular}


Table 4. Hydrogen bond and hydrophobic interaction for the most promising tested compounds into Jak2 binding pocket in comparison to the co-crystallized ligand.

\begin{tabular}{ccccc}
\hline \multirow{2}{*}{ Comp. No } & \multicolumn{3}{c}{ Hydrogen Bonding } & Hydrophobic of Interactions \\
\cline { 2 - 5 } & No & Length Å & AA $^{\text {a }}$ & AA $^{\text {a }}$ \\
\hline 3b & 0 & - & - & LEU855, LEU983, LEU932, VAL863, VAL911, PHE860, ILE982 \\
6c & 1 & 2.940 & ARG980 & LEU855, LEU997, LEU983, LEU932, VAL863, PHE860 \\
6d & 1 & 3.179 & LEU932 & LEU855, LEU997, LEU982, LEU932, VAL863, VAL 911, ILE982 \\
6e & 1 & 2.849 & ARG980 & LEU855, LEU997, LEU983, LEU932, VAL863, VAL 911, ILE982 \\
7c & 2 & 3.181 & PHE860 & LEU884, LEU997, LEU983, LEU932, VAL863, VAL911, PHE860, PHE \\
7d & 1 & 2.264 & ASP976 & 895, ILE982 \\
RL & 2.228 & ARG980 & LEU855, LEU997, LEU983, LEU932, VAL863, VAL911, PHE860, ILE982 \\
& 2 & 3.631 & ARG980 & LEU855, LEU997, LEU983, LEU932, VAL863, VAL911, PHE860, ILE982 \\
\hline
\end{tabular}

Table 5. Hydrogen bond and hydrophobic interaction for the most promising tested compounds into DHFR binding pocket in comparison to the co-crystallized ligand.

\begin{tabular}{|c|c|c|c|c|}
\hline \multirow{3}{*}{ Comp. No } & \multicolumn{4}{|r|}{ Types of Interactions } \\
\hline & \multicolumn{3}{|c|}{ Hydrogen Bonding } & \multirow{2}{*}{$\frac{\text { Hydrophobic }}{\text { AA }^{\mathrm{a}}}$} \\
\hline & No & Length $\AA$ & $\mathbf{A A}^{\mathrm{a}}$ & \\
\hline $3 b$ & 1 & 1.950 & ILE94 & ILE14, ILE5, ILE94, ILE50, LEU24, LEU28, LEU54, PHE31 \\
\hline $6 c$ & 1 & 2.482 & TRP22 & ILE14, ILE5, ILE94, ILE50, LEU24, LEU28, LEU54, LEU8, PHE31 \\
\hline $6 d$ & 2 & $\begin{array}{l}2.482 \\
2.200\end{array}$ & $\begin{array}{c}\text { MET20 } \\
\text { SER49 }\end{array}$ & ILE115, ILE14, ILE5, ILE94, ILE50, LEU24, LEU28, LEU54, LEU8, PHE31 \\
\hline $6 e$ & 1 & 2.476 & SER49 & ILE14, ILE5, ILE94, ILE50, LEU24, LEU28, LEU54, LEU8, PHE31 \\
\hline $7 \mathrm{c}$ & 1 & 2.098 & TRY100 & ILE115, ILE14, ILE5, ILE94, ILE50, LEU24, LEU28, LEU54, LEU8, PHE31 \\
\hline $7 \mathrm{~d}$ & 0 & - & - & ILE14, ILE5, ILE94, ILE50, LEU24, LEU28, LEU54, LEU8, PHE31 \\
\hline Methotrexate & 3 & $\begin{array}{l}2.066 \\
2.135 \\
2.002\end{array}$ & $\begin{array}{l}\text { SER49 } \\
\text { SER49 } \\
\text { THR46 }\end{array}$ & $\begin{array}{l}\text { ILE115, ILE14, ILE5, ILE94, ILE50, LEU24, LEU28, LEU54, LEU8, } \\
\text { VAL13, PHE31 }\end{array}$ \\
\hline
\end{tabular}

RL

A

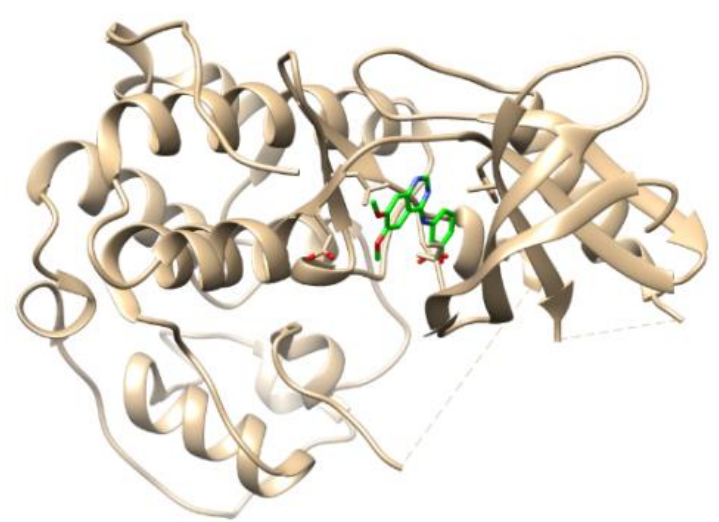

$6 c$

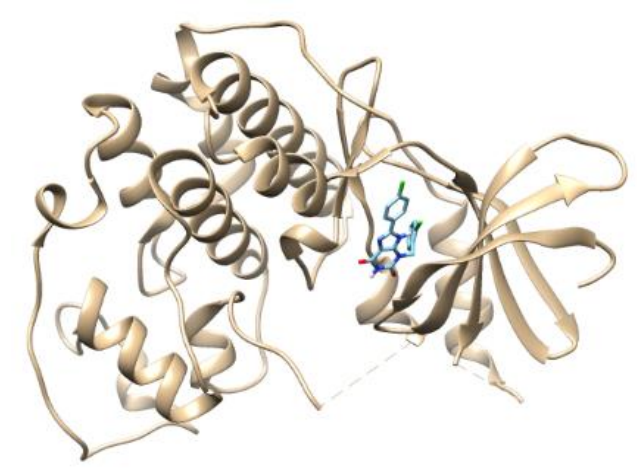

Figure 3. Cont. 
B

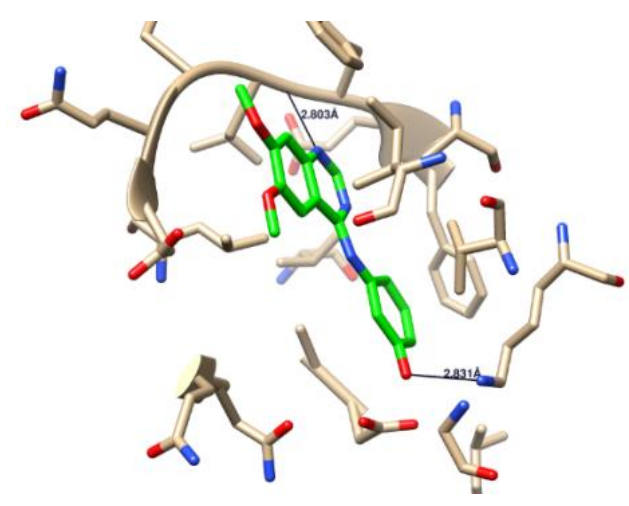

C

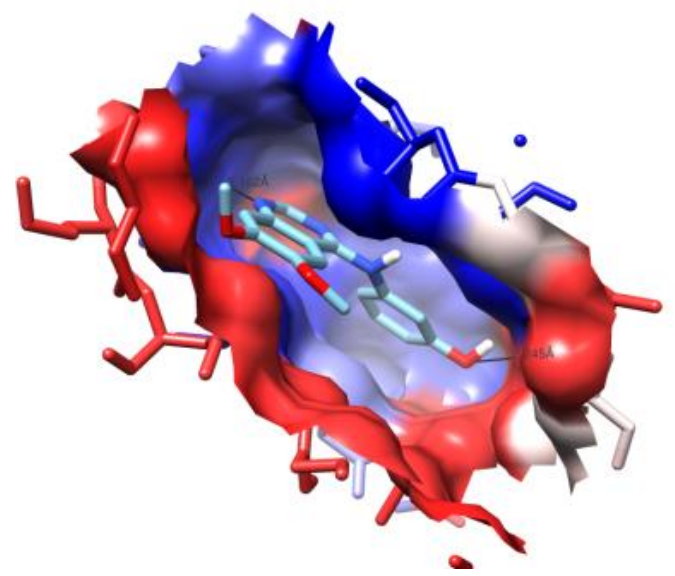

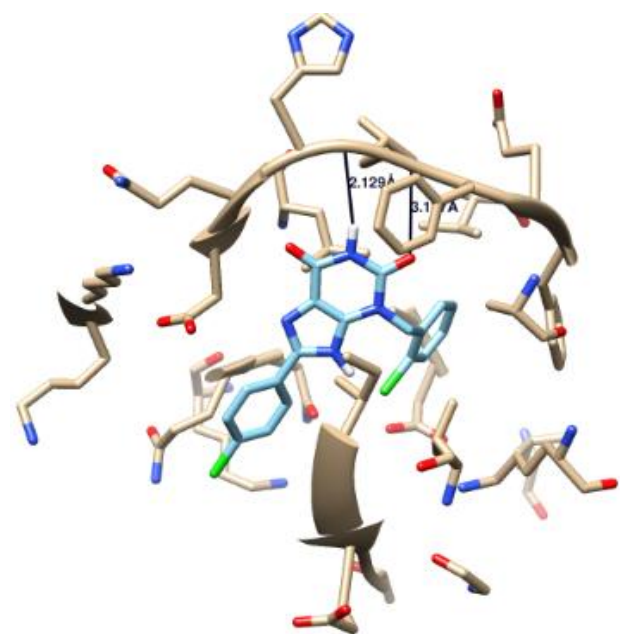

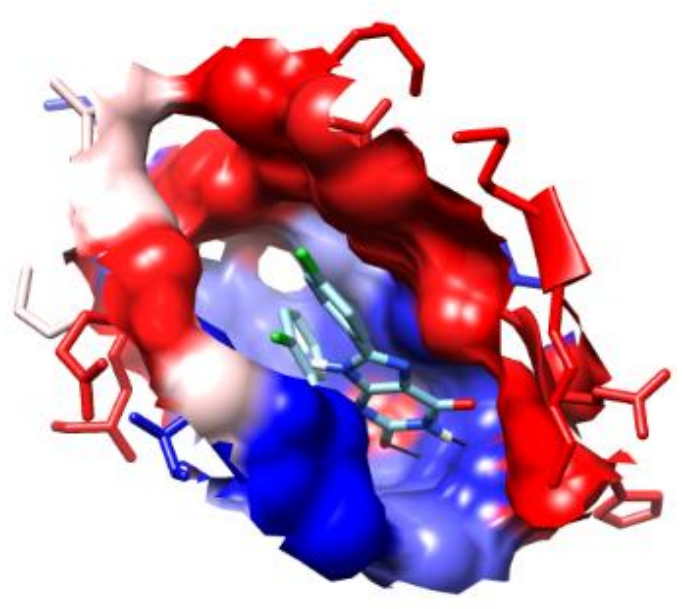

Figure 3. The interaction of compound $\mathbf{6 c}$ with CDK2 protein compared to reference ligand (RL), (A) 3D interaction, (B) hydrogen bond formation, and (C) hydrophobic interaction representation by blue color.

RL

A

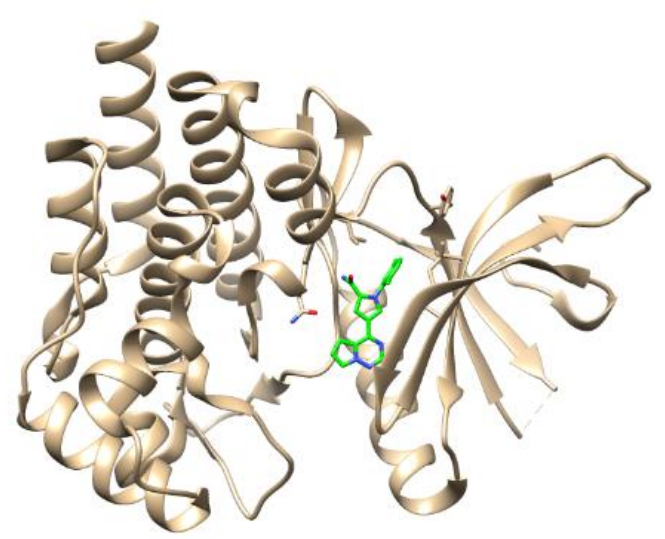

$7 d$

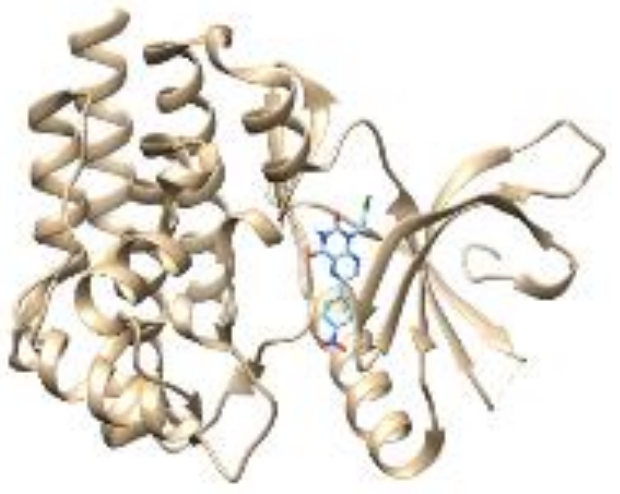

Figure 4. Cont. 
B

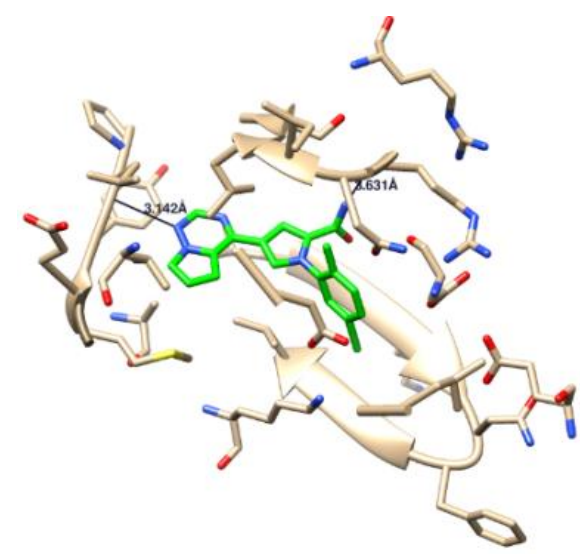

C

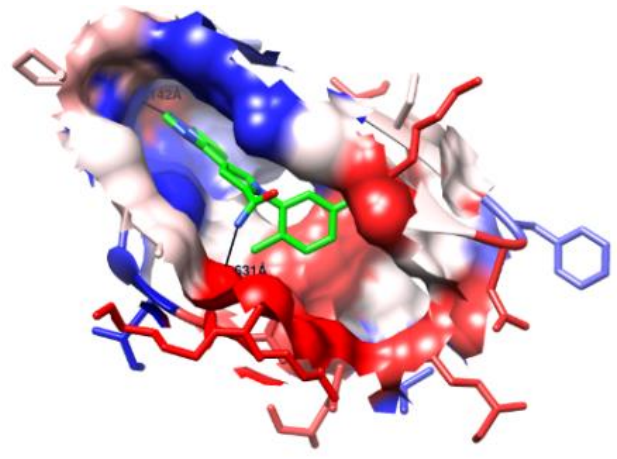

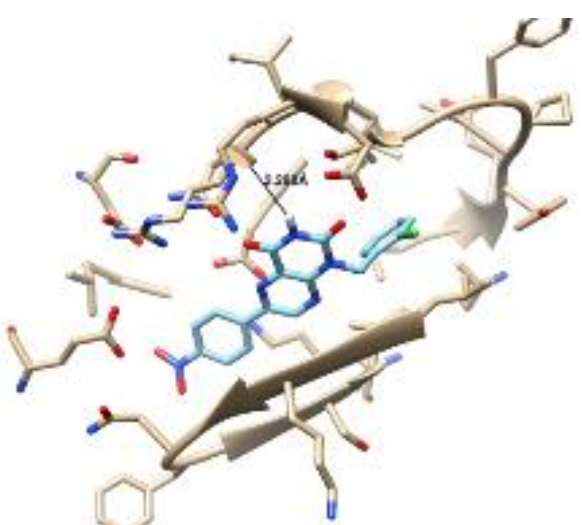

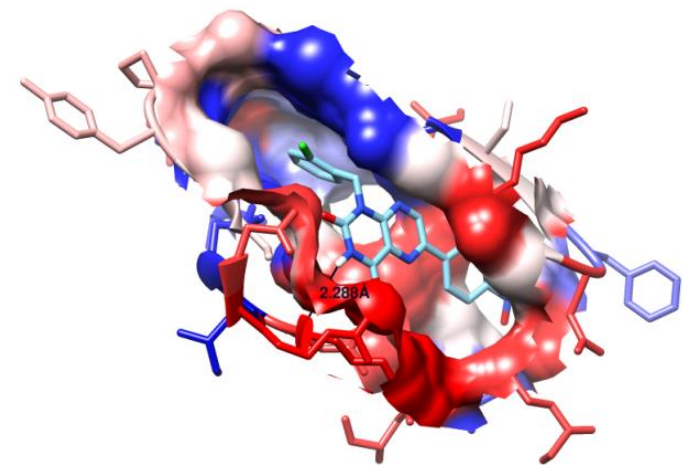

Figure 4. The interaction of compound $7 \mathbf{d}$ with Jak2 protein compared to reference ligand (RL), (A) 3D interaction, (B) hydrogen bond formation, and (C) hydrophobic interaction representation by blue color.

\section{Experimental Part}

\subsection{Chemistry}

All melting points were determined with an Electrothermal Mel.-Temp. II apparatus and were uncorrected. Element analyses were performed at the Regional Center for Mycology and Biotechnology at Al-Azhar University. IR spectra were obtained in the solid state in the form of $\mathrm{KBr}$ discs using a Perkin-Elmer Model 1430 spectrometer (Perkin-Elmer, Akron, OH, USA) and carried out in Taif University, Taif, Saudi Arabia. The proton nuclear magnetic resonance $\left({ }^{1} \mathrm{H}-\mathrm{NMR}\right)$ spectra were recorded on Varian Gemini $400 \mathrm{MHz}$ Spectrometer using DMSO- $d_{6}$ as a solvent (Chemical shift in $\delta$, ppm), Faculty of Science, Chemistry Department, Zagazig University, Zagazig, Egypt. Mass spectra were recorded on a DI-50 unit of Shimadzu GC/MS-QP 5050A (Kyoto, Japan) at the Regional Center for Mycology and Biotechnology at Al-Azhar University, Cairo, Egypt. All reactions were monitored by TLC using precoated plastic sheets silica gel (Merck 60 F254) and spots were visualized by irradiation with UV light $(254 \mathrm{~nm})$. The used solvent system was chloroform: Methanol (9:1) \& ethyl acetate: Toluene (1:1). 6-amino-1-benzyluracil 1a was prepared as the reported method [35], 6-diamino-1-(2-chlorobenzyl)uracil $\mathbf{1 b}$ was prepared as the reported method [36] and 5,6-diamino-1-(2-chlorobenzyl)uracil 4 was prepared as the reported method [36].

\subsubsection{Ethyl 5-Aryl-1-benzyl-7-methyl-2,4-dioxo-1,2,3,4-tetrahydropyrido[2,3-d]pyrimidine-6-carboxylate 3a-d}

General method: A mixture of 6-amino-1-benzyluracil $(0.3 \mathrm{~g}, 1.38 \mathrm{mmol})$, ethyl acetoacetate $(1.38 \mathrm{mmol})$ and appropriate benzaldehyde derivatives $(1.38 \mathrm{mmol})$ in ethanol $(5 \mathrm{~mL})$ in the presence of TEA $(1.0 \mathrm{~mL})$. The reaction mixture was heated under reflux for $12 \mathrm{~h}$. The formed precipitate was filtered off, washed with ethanol and recrystallized from DMF/ethanol (2:1) and dried to give the desired compounds $\mathbf{3 a}-\mathbf{d}$ (Scheme 1). 
Ethyl 1-benzyl-5-phenyl-7-methyl-2,4-dioxo-1,2,3,4-tetrahydropyrido[2,3-d]pyrimidine-6-carboxylate (3a): Yield: 66\%; m.p.: $201-203{ }^{\circ} \mathrm{C}$; IR (KBr) $v_{\max }\left(\mathrm{cm}^{-1}\right)$ : $3186(\mathrm{NH}), 3055$ (CH arom), 2920, 2847 (CH aliph), 1730, 1682 (2C=O), 1595 (NH bending), 694,748 (monosubstituted phenyl); ${ }^{1} \mathrm{H}-\mathrm{NMR}\left(\mathrm{DMSO}-d_{6}\right)$ : $\delta 11.81(\mathrm{~s}, 1 \mathrm{H}, \mathrm{NH}), 7.47-7.43\left(\mathrm{~m}, 5 \mathrm{H}\right.$, arom), 7.30-7.22 (m, 5H, arom), 5.39 (s, 2H, $\left.\mathrm{CH}_{2}\right), 4.15-4.09(\mathrm{q}, 2 \mathrm{H}$, $\left.\mathrm{CH}_{2}\right), 2.72\left(\mathrm{~s}, 3 \mathrm{H}, \mathrm{CH}_{3}(7)\right), 0.99-0.96\left(\mathrm{t}, 3 \mathrm{H}, \mathrm{CH}_{3}\right)$; MS: $m / z(\%)=\mathrm{M}^{+}, 415(100), 400$ (11), 370 (9), $358(8)$, 344 (10), 341 (7), 310 (14), 297 (3), 91 (14), 65 (3); Anal. calcd. for $\mathrm{C}_{24} \mathrm{H}_{21} \mathrm{~N}_{3} \mathrm{O}_{4}$ (415.44): C, 69.39; H, 5.10; $\mathrm{N}, 10.11$. Found: C, 69.13; H, 5.28; N, 10.34 .

Ethyl 1-benzyl-5-(2-chlorophenyl)-7-methyl-2,4-dioxo-1,2,3,4-tetrahydropyrido[2,3-d]pyrimidine-6-carboxylate (3b): Yield: 60\%; m.p.: 258-260 ${ }^{\circ} \mathrm{C}$; IR (KBr) $v_{\max }\left(\mathrm{cm}^{-1}\right): 3165(\mathrm{NH}), 3034(\mathrm{CH}$ arom), 2920, $2840(\mathrm{CH}$ aliph), 1745, 1697 (2C=O), 1603 (NH bending), 754 (o.substituted phenyl); ${ }^{1} \mathrm{H}-\mathrm{NMR}$ (DMSO- $\left.d_{6}\right): \delta 10.92$ (s, 1H, NH), 7.25-7.22 (m, 4H, arom), 7.11-7.03 (m, 3H, arom), 6.87-6.85 (d, 2H, J = 7.2 Hz, arom.), $5.18\left(\mathrm{~s}, 2 \mathrm{H}, \mathrm{CH}_{2}\right), 4.04-3.94\left(\mathrm{q}, 2 \mathrm{H}, \mathrm{J}=6.8, \mathrm{CH}_{2}\right), 2.64\left(\mathrm{~s}, 3 \mathrm{H}, \mathrm{CH}_{3}(7)\right), 1.09-1.06\left(\mathrm{t}, 3 \mathrm{H}, J=6.8, \mathrm{CH}_{3}\right)$; ${ }^{13}$ C-NMR (DMSO- $d_{6}$ ): $\delta=14.02,17.52,43.66,59.55,109.24,125.82,125.92,126.97,127.39,128.17,128.45$, $129.57,129.65,131.32,135.34,137.72,146.17,149.76,151.53,151.56,160.17,165.20 ; \mathrm{MS}: m / z(\%)=\mathrm{M}+2$, 452 (18), $\mathrm{M}^{+}, 450$ (76), 424 (34), 358 (2), 341 (7), 339 (7), 258 (3), 152 (2), 92 (5), 91 (100), 65 (11); Anal. calcd. for $\mathrm{C}_{24} \mathrm{H}_{20} \mathrm{ClN}_{3} \mathrm{O}_{4}$ (449.88): C, 64.07; H, 4.48; N, 9.34. Found: C, 63.89; H, 4.62; N, 9.51.

Ethyl 1-benzyl-5-(4-bromophenyl)-7-methyl-2,4-dioxo-1,2,3,4-tetrahydropyrido[2,3-d]pyrimidine-6-carboxylate (3c): Yield: 73\%; m.p.: 217-219 ${ }^{\circ} \mathrm{C}$; IR (KBr) $v_{\max }\left(\mathrm{cm}^{-1}\right): 3117(\mathrm{NH}), 3031$ (CH arom), 2948, 2845 (CH aliph), 1725, $1681(2 \mathrm{C}=\mathrm{O}), 1558$ (NH bending), 838 ( $p$.substituted phenyl); ${ }^{1} \mathrm{H}-\mathrm{NMR}$ (DMSO- $\left.d_{6}\right): \delta 11.82$ (s, 1H, NH), 7.70-7.68 (d, 2H, J = 8.4 Hz, arom), 7.38-7.36 (d, 2H, J = 8.4 Hz, arom), 7.30-7.29 (d, 2H, $J=4.4 \mathrm{~Hz}$, arom.), $7.23-7.18\left(\mathrm{~m}, 3 \mathrm{H}\right.$, arom), $5.3\left(\mathrm{~s}, 2 \mathrm{H}, \mathrm{CH}_{2}\right), 4.19-4.13\left(\mathrm{q}, 2 \mathrm{H}, J=6.8, \mathrm{CH}_{2}\right), 2.73(\mathrm{~s}, 3 \mathrm{H}$, $\left.\mathrm{CH}_{3}(7)\right), 1.05-1.02(\mathrm{t}, 3 \mathrm{H}, J=6.8, \mathrm{CH} 3) ;{ }^{13} \mathrm{C}-\mathrm{NMR}\left(\mathrm{DMSO}-d_{6}\right): \delta=13.43,18.03,44.62,61.78,108.26$, $123.65,125.39,126.95,127.14,128.29,130.01,131.61,137.26,137.44,150.30,150.92,151.87,156.08,161.53$, 167.32; MS: $m / z(\%)=M+2,496$ (12), $\mathrm{M}^{+}, 494$ (32), 490 (23), 387 (34), 341 (11), 292 (59), 265 (62), 129 (58), 72 (100), 69 (92); Anal. calcd. for $\mathrm{C}_{24} \mathrm{H}_{20} \mathrm{ClN}_{3} \mathrm{O}_{4}$ (494.33): C, 58.31; H, 4.08; N, 8.50. Found: C, 58.57; $\mathrm{H}, 4.23 ; \mathrm{N}, 8.69$.

Ethyl 1-benzyl-7-methyl-5-(3-nitrophenyl)-2,4-dioxo-1,2,3,4-tetrahydropyrido[2,3-d]pyrimidine-6-carboxylate (3d): Yield: 84\%; m.p.: 190-192 ${ }^{\circ} \mathrm{C}$; IR (KBr) $v_{\max }\left(\mathrm{cm}^{-1}\right): 3215(\mathrm{NH}), 3050$ (CH arom), 2965, $2869(\mathrm{CH}$ aliph), 1723, $1684(2 \mathrm{C}=\mathrm{O}), 1614(\mathrm{C}=\mathrm{C}), 1551,1374\left(\mathrm{NO}_{2}\right), 755$ (m.substituted phenyl); ${ }^{1} \mathrm{H}-\mathrm{NMR}$ $\left(\mathrm{DMSO}-d_{6}\right): \delta 11.90$ (bs, 1H, NH), 8.25 (s, 1H, arom), 7.94-7.39 (m, 3H, arom), 7.32-7.16 (m, 5H, arom), $5.24\left(\mathrm{~s}, 2 \mathrm{H}, \mathrm{CH}_{2}\right), 4.18-4.14\left(\mathrm{q}, 2 \mathrm{H}, \mathrm{J}=7.2 \mathrm{~Hz}, \mathrm{CH}_{2}\right), 2.88\left(\mathrm{~s}, 3 \mathrm{H}, \mathrm{CH}_{3}(7)\right), 1.04-1.01(\mathrm{t}, 3 \mathrm{H}$, $\left.J=7.2 \mathrm{~Hz}, \mathrm{CH}_{3}\right) ;{ }^{13} \mathrm{C}-\mathrm{NMR}\left(\mathrm{DMSO}-d_{6}\right): \delta=13.41,18.13,44.78,61.81,107.90,123.18,125.23,126.94$, 127.03, 127.22, 127.59, 128.29, 131.61, 136.43, 137.26, 137.44, 150.50, 150.98, 151.84, 156.12, 161.60, 167.45; MS: $m / z(\%)=\mathrm{M}^{+}, 460$ (13), 430 (70), 391 (51), 360 (27), 345 (28), 343 (18), 234 (31), 209 (64), 182 (29), 173 (100), 172 (29), 69 (92); Anal. calcd. for $\mathrm{C}_{24} \mathrm{H}_{20} \mathrm{~N}_{4} \mathrm{O}_{6}$ (460.43): C, 62.60; H, 4.38; N, 12.17. Found: C, 62.86; H, 4.54; N, 12.41 .

6-Acetyl-1-benzyl-5-(2-hydroxyphenyl)pyrido[2,3-d]pyrimidine-2,4,7(1H,3H,8H)-trione (3e): A mixture of 6-amino-1-benzyluracil ( $0.5 \mathrm{~g}, 2.3 \mathrm{mmol})$, ethyl 2-(2-hydroxybenzylidene) acetoacetate $(2.3 \mathrm{mmol})$ was mixed in DMF $(1.5 \mathrm{~mL})$ in the presence of TEA $(1.0 \mathrm{~mL})$. The reaction mixture was heated under reflux for $7 \mathrm{~h}$. The mixture was evaporated in vacuo and the residue was treated with methanol $(10 \mathrm{~mL})$. The formed precipitate was filtered off and recrystallized from DMF/ethanol (2:1) to afford 3e. Yield: $68 \%$; m.p.: $260-262{ }^{\circ} \mathrm{C}$; IR (KBr) $v_{\max }\left(\mathrm{cm}^{-1}\right): 3448(\mathrm{OH}), 3186(\mathrm{NH}), 3047(\mathrm{CH}$ arom), 2924, 2846 (CH aliph), 1681 (C=O), 1589 (NH bending), 763 (o.substituted phenyl); ${ }^{1} \mathrm{H}-\mathrm{NMR}$ (DMSO- $\left.d_{6}\right)$ : $\delta 13.98$ (s, 1H, OH), 11.98 (s, 1H, NH), 8.19-8.17 (d, 1H, arom), 7.69 (s, 1H, NH 8), 7.67-7.61 (m, 3H, arom), 7.43-7.21 (m, 5H, arom), $5.45(\mathrm{~s}, 2 \mathrm{H}, \mathrm{CH} 2), 2.90\left(\mathrm{~s}, 3 \mathrm{H}, \mathrm{CH}_{3}(6)\right) ;{ }^{13} \mathrm{C}-\mathrm{NMR}\left(\mathrm{DMSO}-d_{6}\right): \delta=17.52$, 43.66, 103.73, 112.40, 116.16, 122.65, 124.73, 127.10, 127.75, 128.33, 132.03, 133.62, 137.22, 146.90, 150.29, 151.89, 153.86, 158.19, 160.37, 167.14; MS: $m / z(\%)=\mathrm{M}^{+}, 403$ (84), $361(29), 315$ (24), 268 (19), 250 (100), 228 (23), 186 (48), 125 (87), 76 (53), 56 (30); Anal. calcd. for $\mathrm{C}_{22} \mathrm{H}_{17} \mathrm{~N}_{3} \mathrm{O}_{5}$ (403.38): C, 65.50; H, 4.25; N, 10.42. Found: C, 65.89; H, 4.32; N, 10.51. 
6-Amino-1-(2-chlorobenzyl)-5-[(E)-(4-chlorobenzylidene)amino]pyrimidine-2,4(1H,3H)-dione (5): A mixture of 5,6-diamino-1-(2-chlorobenzyl)uracil $(0.8 \mathrm{~g}, 2.0 \mathrm{mmol})$, 4-chlorobenzaldehyde $(0.2 \mathrm{mmol})$ in DMF $(3.0 \mathrm{~mL})$ was heated under reflux for $45 \mathrm{~min}$. After cooling, the formed precipitate was collected by filtration, washed with ethanol and dried in an oven to give $(1.01 \mathrm{~g})$. Yield: $87 \%$; m.p.: 283-285 ${ }^{\circ} \mathrm{C}$; IR $(\mathrm{KBr}) v_{\max }\left(\mathrm{cm}^{-1}\right)$ : 3339, $3274\left(\mathrm{NH}_{2}\right), 3184(\mathrm{NH}), 3078(\mathrm{CH}$ arom $), 1691,1643(2 \mathrm{C}=\mathrm{O})$, 822 (p.substituted phenyl), $748(\mathrm{C}-\mathrm{Cl}) ;{ }^{1} \mathrm{H}-\mathrm{NMR}\left(\mathrm{DMSO}-d_{6}\right): \delta 10.92(\mathrm{~s}, 1 \mathrm{H}, \mathrm{NH} 3), 9.72(\mathrm{~s}, 1 \mathrm{H}, \mathrm{CH})$, $7.52-7.50(\mathrm{~d}, 2 \mathrm{H}, J=8.4 \mathrm{~Hz}$, arom.), 7.44-7.34 (m, 4H, arom), 7.27-7.25 (d, 2H, $J=8.4 \mathrm{~Hz}$, arom), 6.99 (s, 2H, NH $\mathrm{NH}_{2}$ ), 5.24 (s, 2H, $\left.\mathrm{CH}_{2}\right)$; MS: $m / z(\%)=\mathrm{M}+2,391(4), \mathrm{M}^{+}, 389$ (14), 375 (5), 263 (10), 246 (92), 216 (30), 190 (13), 125 (100), 89 (59), 63 (18); Anal. calcd. for $\mathrm{C}_{18} \mathrm{H}_{14} \mathrm{Cl}_{2} \mathrm{~N}_{4} \mathrm{O}_{2}$ (389.23): C, 55.54; $\mathrm{H}, 3.63 ; \mathrm{N}, 14.39$. Found: C, 55.62; H, 3.65; N, 14.53 .

\subsubsection{8-Aryl-3-(2-chlorobenzyl)-xanthine}

8-Aryl-3-(2-chlorobenzyl)-3,9-dihydro-1H-purine-2,6-dione 6a-f

General method: Method A: To 5,6-diamino-1-(2-chlorobenzyl)uracil 4 (0.5 mmol), was added different aromatic aldehydes $(0.5 \mathrm{mmol})$ in DMF $(3.0 \mathrm{~mL})$. The reaction mixture was heated under reflux for 2-3 h. After cooling, the formed precipitate was filtered off, washed with methanol and recrystallized from $\mathrm{DMF} / \mathrm{H}_{2} \mathrm{O}$ 3:1) to give the desired compounds $\mathbf{6 a}-\mathbf{f}$ in good yields. Method B: Compound $5(0.2 \mathrm{~g}, 0.5 \mathrm{mmol})$ in DMF $(3 \mathrm{~mL})$ was heated under reflux for $90 \mathrm{~min}$. After cooling, the formed precipitate was collected by filtration, washed with methanol and dried in an oven to afford $6 \mathrm{c}$ in a good yield.

\subsubsection{3-(2-Chlorobenzyl)-8-phenylxanthine}

3-(2-chlorobenzyl)-8-phenyl-3,9-dihydro-1H-purine-2,6-dione (6a): Yield 83\%; m.p.: $>300{ }^{\circ} \mathrm{C}$; IR (KBr) $v_{\max }$ $\left(\mathrm{cm}^{-1}\right)$ : 3334, 3224 (2NH), 3078 (CH arom), 2970, 2831 (CH aliph), 1667 (C=O), 748 (C-Cl); ${ }^{1} \mathrm{H}-\mathrm{NMR}$ (DMSO- $\left.d_{6}\right): \delta 13.23(\mathrm{~s}, 1 \mathrm{H}, \mathrm{NH}$ 9), $10.94(\mathrm{~s}, 1 \mathrm{H}, \mathrm{NH} 1), 7.95-7.61(\mathrm{~m}, 2 \mathrm{H}$, arom.), 7.51-7.48 (m, 3H, arom), 7.35-7.32 (m, 3H, arom), 6.88-6.84 (m, $1 \mathrm{H}$, arom), $5.07\left(\mathrm{~s}, 2 \mathrm{H}, \mathrm{CH}_{2}\right) ;{ }^{13} \mathrm{C}-\mathrm{NMR}$ (DMSO- $\left.d_{6}\right): \delta=43.0$, $107.80,125.39,127.49,127.50,128.60,128.69,129.38,131.46,131.61,133.31,133.69,149.78,149.99,154.62$, 162.29; MS: $m / z(\%)=\mathrm{M}+2,354$ (4), $\mathrm{M}^{+}, 352$ (16), 331 (26), 312 (29), 286 (30), 239 (29), 110 (28), 98 (31), 84 (35), 83 (100); Anal. calcd. for $\mathrm{C}_{18} \mathrm{H}_{13} \mathrm{ClN}_{4} \mathrm{O}_{2}$ (352.78): $\mathrm{C}, 61.28 ; \mathrm{H}, 3.71 ; \mathrm{N}, 15.88$. Found: $\mathrm{C}, 61.47$; $\mathrm{H}, 3.87 ; \mathrm{N}, 15.74$.

3-[(2-Chlorophenyl)methyl]-8-(4-hydroxyphenyl)-3,9-dihydro-1H-purine-2,6-dione

3-(2-Chlorobenzyl)-8-(4-hydroxyphenyl)xanthine (6b):Yield: 57\%; m.p.: $>300{ }^{\circ} \mathrm{C}$; IR (KBr) $v_{\max }\left(\mathrm{cm}^{-1}\right)$ : $3450(\mathrm{OH}), 3330,3142$ (2NH), 3027 (CH arom), 2922, 2819 (CH aliph.), 1667 (C=O), 1599 (NH bending), 841 (p. substituted phenyl), 766. (C-Cl); ${ }^{1} \mathrm{H}-\mathrm{NMR}$ (DMSO- $\left.d_{6}\right): \delta 13.55(\mathrm{~s}, 1 \mathrm{H}, \mathrm{NH} 9), 11.21(\mathrm{~s}, 1 \mathrm{H}$, $\mathrm{NH}$ 1), $9.96(\mathrm{~s}, 1 \mathrm{H}, \mathrm{OH}), 7.89-7.87(\mathrm{~d}, 2 \mathrm{H}, J=6.8 \mathrm{~Hz}$, arom. $), 7.31-7.25(\mathrm{~m}, 3 \mathrm{H}$, arom $), 7.04-7.02(\mathrm{~m}, 1 \mathrm{H}$, arom), 6.83-6.81 (d, $2 \mathrm{H}, J=6.8$, arom), $5.23\left(\mathrm{~s}, 2 \mathrm{H}, \mathrm{CH}_{2}\right) ;{ }^{13} \mathrm{C}-\mathrm{NMR}\left(\mathrm{DMSO}-d_{6}\right): \delta=43.0,107.6,115.7$, 119.57, 127.06, 127.48, 128.21, 128.78, 129.34, 131.51, 134.15, 149.87, 150.95, 154.62, 159.45, 163,29; MS: $m / z(\%)=M+2,370(2), \mathrm{M}^{+}, 368(4), 333$ (22), 259 (24), 216 (12), 125 (100), 89 (26), 77 (3); Anal. calcd. for $\mathrm{C}_{18} \mathrm{H}_{13} \mathrm{ClN}_{4} \mathrm{O}_{3}$ (368.77): C, 58.62; $\mathrm{H}, 3.55 ; \mathrm{N}$, 15.19. Found: $\mathrm{C}, 58.74 ; \mathrm{H}, 3.58 ; \mathrm{N}, 15.34$.

3-[(2-Chlorophenyl)methyl]-8-(4-chlorophenyl)-3,9-dihydro-1H-purine-2,6-dione

3-(2-Chlorobenzyl)-8-(4-chlorophenyl)xanthine (6c): Method A: Yield: 67\% Method B: Yield: 85\%; m.p.: $>300^{\circ} \mathrm{C}$; IR (KBr) $v_{\max }\left(\mathrm{cm}^{-1}\right): 3311,3142(2 \mathrm{NH}), 3022(\mathrm{CH}$ arom), 2987, 2838 ( $\mathrm{CH}$ aliph), $1682(\mathrm{C}=\mathrm{O})$, 1583 (NH bending), 830 (p.substituted phenyl), 740 (C-Cl); ${ }^{1} \mathrm{H}-\mathrm{NMR}$ (DMSO- $\left.d_{6}\right): \delta 13.99(\mathrm{~s}, 1 \mathrm{H}, \mathrm{NH} 9)$, $11.33(\mathrm{~s}, 1 \mathrm{H}, \mathrm{NH}$ 1), 8.06-8.04 (d, 2H, $J=6.8 \mathrm{~Hz}$, arom), 7.55-7.53 (d, 2H, $J=6.8 \mathrm{~Hz}$, arom), 7.30-7.07 (m, 3H, arom.), $7.06-7.05\left(\mathrm{~d}, 1 \mathrm{H}, J=1.6 \mathrm{~Hz}\right.$, arom), $5.24\left(\mathrm{~s}, 2 \mathrm{H}, \mathrm{CH}_{2}\right) ;{ }^{13} \mathrm{C}-\mathrm{NMR}$ (DMSO- $\left.d_{6}\right): \delta=43.06$, 108.6, 127.11, 127.46, 128.02, 128.79, 129.08, 129.33, 131.49, 134.00, 134.83, 149.65, 150.89, 150.98, 154.69, 163.20; MS: $m / z(\%)=\mathrm{M}+4,391$ (0.25), $\mathrm{M}+2,389$ (0.75), $\mathrm{M}^{+}, 387$ (2), 353 (13), 351 (45), 175 (4), 138 (11), 
127 (26), 125 (100), 89 (24); Anal. calcd. for $\mathrm{C}_{18} \mathrm{H}_{12} \mathrm{Cl}_{2} \mathrm{~N}_{4} \mathrm{O}_{2}$ (387.21): C, 55.83; H, 3.12; $\mathrm{N}, 14.47$. Found: C, $56.01 ; \mathrm{H}, 3.19 ; \mathrm{N}, 14.62$.

3-[(2-Chlorophenyl)methyl]-8-(2-hydroxyphenyl)-3,9-dihydro-1H-purine-2,6-dione

3-(2-Chlorobenzyl)-8-(2-hydroxyphenyl)xanthine (6d): Yield: 58\%; m.p.: >300; IR (KBr) $v_{\max }\left(\mathrm{cm}^{-1}\right)$ : $3480(\mathrm{OH}), 3320,3168(2 \mathrm{NH}), 3026$ (CH arom), 2910, 2831 (CH aliph,), 1668, $1643(2 \mathrm{C}=\mathrm{O}), 1583(\mathrm{NH}$ bending), 747 (o.substituted phenyl); ${ }^{1} \mathrm{H}-\mathrm{NMR}$ (DMSO- $\left.d_{6}\right): \delta 13.85$ (s, $1 \mathrm{H}, \mathrm{NH}$ 9), $11.41(\mathrm{~s}, 1 \mathrm{H}, \mathrm{NH} 1)$, 9.55 (s, 1H, OH), 8.01-7.99 (d, 1H, arom.), 7.52-7.50 (m, 2H, arom), 7.31-7.25 (m, 3H, arom), 7.17-6.90 $\left(\mathrm{m}, 2 \mathrm{H}\right.$, arom), $5.26\left(\mathrm{~s}, 2 \mathrm{H}, \mathrm{CH}_{2}\right) ;{ }^{13} \mathrm{C}-\mathrm{NMR}$ (DMSO- $\left.d_{6}\right): \delta=42.30(\mathrm{CH} 2), 108.10,123.00,125.20,127.25$, $127.55,127.75,128.16,129.07,129.38,129.49,131.30,134.28,149.79,150.96,154.21,161.67,162.33$; MS: $m / z(\%)=M+2,370(2), \mathrm{M}^{+}, 368(0.98), 290(4), 259$ (34), 172 (21), 152 (5), 125 (100), 107 (16), 89 (28), 77 (11); Anal. calcd. for $\mathrm{C}_{18} \mathrm{H}_{13} \mathrm{ClN}_{4} \mathrm{O}_{3}$ (368.77): C, 58.62; $\mathrm{H}, 3.55 ; \mathrm{N}, 15.19$. Found: $\mathrm{C}, 58.72 ; \mathrm{H}, 3.59$; $\mathrm{N}, 15.31$.

3-[(2-Chlorophenyl)methyl]-8-(4-nitrophenyl)-3,9-dihydro-1H-purine-2,6-dione

3-(2-Chlorobenzyl)-8-(4-nitrophenyl)xanthine (6e): Yield: 71\%; m.p.: >300; IR (KBr) $v_{\max }\left(\mathrm{cm}^{-1}\right)$ : 3329, 3155 (2NH), 3042 ( $\mathrm{CH}$ arom), 2926, 2822 ( $\mathrm{CH}$ aliph), 1685, 1664 (2C=O), 1573 (NH bending), 1518, $1338\left(\mathrm{NO}_{2}\right), 854$ (p.substituted phenyl), $745(\mathrm{C}-\mathrm{Cl}) ;{ }^{1} \mathrm{H}-\mathrm{NMR}$ (DMSO- $\left.d_{6}\right): \delta 14.36(\mathrm{~s}, 1 \mathrm{H}, \mathrm{NH} 9)$, $11.39(\mathrm{~s}, 1 \mathrm{H}, \mathrm{NH}), 8.32-8.30(\mathrm{~d}, 2 \mathrm{H}, J=8.4 \mathrm{~Hz}$, arom), 7.52-7.50 (d, 2H, $J=8.4 \mathrm{~Hz}$, arom), 7.33-7.25 (m, 3H, arom), 7.09-7.07 (d, 3H, $J=7.2$, arom.), $5.25\left(\mathrm{~s}, 2 \mathrm{H}, \mathrm{CH}_{2}\right) ;{ }^{13} \mathrm{C}-\mathrm{NMR}$ (DMSO- $d_{6}$ ): $\delta=43.45$, 107.90, 124.25, 127.12, 127.46, 128.06, 128.30, 128.90, 129.34, 131.50, 134.82, 149.50, 150.90, 152.0, 154.23, 162.21; MS: $m / z(\%)=\mathrm{M}+2,399(0.46), \mathrm{M}^{+}, 397$ (0.7), 324 (29), 283 (6), 166 (30), 150 (10), 127 (100), 91 (18), 67 (17); Anal. calcd. for $\mathrm{C}_{18} \mathrm{H}_{12} \mathrm{ClN}_{5} \mathrm{O}_{4}$ (397.21): C, 54.35; H, 3.04; N, 17.61. Found: C, 54.52; $\mathrm{H}, 3.12 ; \mathrm{N}, 17.89$.

8-(4-Bromophenyl)-3-[(2-chlorophenyl)methyl]-3,9-dihydro-1H-purine-2,6-dione

8-(4-Bromophenyl)-3-(2-Chlorobenzyl)xanthine (6f): Yield: $62 \%$; m.p.: $>300^{\circ} \mathrm{C} ; \mathrm{IR}(\mathrm{KBr}) v_{\max }\left(\mathrm{cm}^{-1}\right)$ : 3280, 3170 (2NH), 3091 (CH arom), 2998, 2802 (CH aliph), 1678, (C=O), 1583 (NH bending), 824 (p.substituted), 741 (C-Cl), 660 (C-Br); ${ }^{1} \mathrm{H}-\mathrm{NMR}\left(\mathrm{DMSO}-d_{6}\right): \delta 14.03$ (s, 1H, NH 9), 11.35 (s, 1H, NH), 7.99-7.97 (d, 2H, $J=8.4 \mathrm{~Hz}$, arom), 7.69-7.67 (d, 2H, $J=8.4 \mathrm{~Hz}$, arom), 7.32-7.23 (m, 3H, arom.), 7.07-7.05 (d, 1H, $J=7.2 \mathrm{~Hz}$, arom.), $5.23\left(\mathrm{~s}, 2 \mathrm{H}, \mathrm{CH}_{2}\right) ;{ }^{13} \mathrm{C}-\mathrm{NMR}\left(\mathrm{DMSO}-d_{6}\right): \delta=42.90,108.60,127.08,127.44,128.17$, $128.77,130.69,131.48,131.94,134.03,136.24,149.70,150.90,154.72,157.32,162.30 ; \mathrm{MS}: \mathrm{m} / z(\%)=\mathrm{M}+4$, 435 (0.87), M + 2, 433 (0.79), M+ , 431 (5), 394 (5), 308 (14), 216 (13), 169 (19), 73 (100), 43 (12); Anal. calcd. for $\mathrm{C}_{18} \mathrm{H}_{12} \mathrm{BrClN}_{4} \mathrm{O}_{2}$ (431.67): $\mathrm{C}, 50.08 ; \mathrm{H}, 2.80 ; \mathrm{N}, 12.98$. Found: $\mathrm{C}, 50.19 ; \mathrm{H}, 2.77 ; \mathrm{N}, 13.14$.

6-Aryl-1-[(2-chlorophenyl)methyl]pteridine-2,4(1H,3H)-dione

6-Aryl-1-(2-chlorobenzyl)lumazine 7a-d: General method: Method A: A mixture of 5,6-diaminouracil 4 $(0.5 \mathrm{mmol})$, appropriate aromatic aldehydes $(0.5 \mathrm{mmol})$ and triethyl orthoformate $(4.5 \mathrm{~mL})$ was heated under reflux for $7-8 \mathrm{~h}$. After cooling, the formed precipitate was collected by filtration, recrystallized from DMF/EtOH (2:1). Method B: Compound $5(0.2 \mathrm{~g}, 0.5 \mathrm{mmol})$ in triethyl orthoformate $(5 \mathrm{~mL})$ was heated under reflux for $4 \mathrm{~h}$. After cooling, the formed precipitate was collected by filtration, washed with methanol and dried in an oven to afford $7 \mathbf{a}-\mathbf{d}$.

6-(4-Chlorophenyl)-1-[(2-chlorophenyl)methyl]pteridine-2,4(1H,3H)-dione

6-(4-Chlorophenyl)-1-(2-chlorobenzyl)lumazine (7a): Method A: Yield: 74\%, Method B: Yield: 82\%; m.p.: 250-252 ${ }^{\circ} \mathrm{C}$; IR (KBr) $v_{\max }\left(\mathrm{cm}^{-1}\right): 3141(\mathrm{NH}), 3070$ (CH arom), 2976, 2860 ( $\mathrm{CH}$ aliph), 1682, $1668(2 \mathrm{C}=\mathrm{O})$, 1590 (NH bending), 898 ( $p$.substituted phenyl), 748 (C-Cl); ${ }^{1} \mathrm{H}-\mathrm{NMR}$ (DMSO- $d_{6}$ ): $\delta 11.99(\mathrm{~s}, 1 \mathrm{H}, \mathrm{NH})$, $8.71(\mathrm{~s}, 1 \mathrm{H}, \mathrm{CH}-7), 7.28-7.26(\mathrm{~d}, 2 \mathrm{H}, J=8.0 \mathrm{~Hz}$, arom), 7.11-6.96 (m, 4H, arom), 6.84-6.82 (d, 2H, $J=8.0 \mathrm{~Hz}$, arom), $5.10\left(\mathrm{~s}, 2 \mathrm{H}, \mathrm{CH}_{2}\right) ;{ }^{13} \mathrm{C}-\mathrm{NMR}\left(\mathrm{DMSO}_{-} \mathrm{d}_{6}\right): \delta=42.46,107.28,126.09,127.45,128.24,128.58$, 
$129.21,129.35,131.09,131.48,133.90,137.61,150.39,151.12,154.11,155.89,162.33 ; \mathrm{MS}: \mathrm{m} / \mathrm{z}(\%)=\mathrm{M}+4$, 403 (0.70), M + 2, 401, (1), M+ 399 (2.45), 328 (3), 190 (25), 122 (100), 93(46), 65 (48); Anal. calcd. for $\mathrm{C}_{19} \mathrm{H}_{12} \mathrm{Cl}_{2} \mathrm{~N}_{4} \mathrm{O}_{2}$ (399.23): C, 57.16; $\mathrm{H}, 3.03 ; \mathrm{N}, 14.03$. Found: $\mathrm{C}, 57.34 ; \mathrm{H}, 3.11 ; \mathrm{N}, 14.29$.

1-[(2-Chlorophenyl)methyl]-6-(4-hydroxyphenyl)pteridine-2,4(1H,3H)-dione

1-(2-chlorobenzyl)-6-(4-hydroxyphenyl)lumazine (7b): Yield: 57\%; m.p.: $280-282{ }^{\circ} \mathrm{C}$; IR $(\mathrm{KBr}) v_{\max }\left(\mathrm{cm}^{-1}\right)$ : $3480(\mathrm{OH}), 3127(\mathrm{NH}), 3025(\mathrm{CH}$ arom), 2914, 2856 (CH aliph), 1697, $1654(2 \mathrm{C}=\mathrm{O}), 1609(\mathrm{C}=\mathrm{C})$, 827 (p.substituted phenyl), $749(\mathrm{C}-\mathrm{Cl}) ;{ }^{1} \mathrm{H}-\mathrm{NMR}\left(\mathrm{DMSO}-d_{6}\right): \delta 12.01(\mathrm{~s}, 1 \mathrm{H}, \mathrm{NH}), 10.53(\mathrm{~s}, 1 \mathrm{H}, \mathrm{OH})$, 8.71 (s, 1H, CH-7), 7.34-7.27 (m, 3H, arom), 7.13-7.09 (t, 1H, arom), 6.99-6.95 (t, 1H, arom), 6.87-6.83 (m, 3H, arom.), $5.10\left(\mathrm{~s}, 2 \mathrm{H}, \mathrm{CH}_{2}\right) ;{ }^{13} \mathrm{C}-\mathrm{NMR}$ (DMSO- $\left.d_{6}\right): \delta=42.97,107.29,125.35,126.86,127.45,128.25$, $128.94,129.35,131.49,133.90,137.62,150.39,151.12,154.11,155.89,160.27,162.34 ; \mathrm{MS}: \mathrm{m} / \mathrm{z}(\%)=\mathrm{M}+2$, 382 (0.58), M $\mathrm{M}^{+}, 380$ (0.18), 281 (4), 270 (3), 253 (4), 242 (16), 241 (100), 210 (8), 198 (16), 125 (98), 99 (14), 89 (31), 71 (19); Anal. calcd. for $\mathrm{C}_{19} \mathrm{H}_{13} \mathrm{ClN}_{4} \mathrm{O}_{3}$ (380.78): C, 59.93; H, 3.44; N, 14.71. Found: C, 60.12; $\mathrm{H}, 3.49 ; \mathrm{N}, 14.87$.

1-(2-Chlorobenzyl)-6-(2-hydroxyphenyl)pteridine-2,4(1H,3H)-dione

1-(2-Chlorobenzyl)-6-(2-hydroxyphenyl)lumazine (7c): Yield: 61\%; m.p.: 252-254 ${ }^{\circ} \mathrm{C} ; \mathrm{IR}(\mathrm{KBr}) v_{\max }\left(\mathrm{cm}^{-1}\right)$ : $3423(\mathrm{OH}), 3179(\mathrm{NH}), 3051$ ( $\mathrm{CH}$ arom), 2911, 2850 ( $\mathrm{CH}$ aliph), $1685(\mathrm{C}=\mathrm{O}), 1583$ (NH bending), 761 (o.substituted phenyl); ${ }^{1} \mathrm{H}-\mathrm{NMR}$ (DMSO- $\left.d_{6}\right): \delta 11.29(\mathrm{~s}, 1 \mathrm{H}, \mathrm{NH}), 10.25(\mathrm{~s}, 1 \mathrm{H}, \mathrm{OH}), 8.70(\mathrm{~s}, 1 \mathrm{H}$, CH-7), 7.66-7.64 (d, 1H, arom), 7.53-7.50 (t, 1H, arom), 7.31-7.22 (m, 3H, arom.), 7.18-7.00 (m, 1H, arom), 6.98-6.82 (m, 2H, arom.), 5.09 (s, 2H, CH2); MS: $m / z(\%)=\mathrm{M}+2,382$ (17), $\mathrm{M}^{+}, 380$ (19), 359 (37), 354 (60), 229 (100), 217 (50), 177 (24), 83 (21), 60 (42); Anal. calcd. for $\mathrm{C}_{19} \mathrm{H}_{13} \mathrm{ClN}_{4} \mathrm{O}_{3}$ (380.78): C, 59.93; H, 3.44; N, 14.71. Found: C, 60.19; H, 3.57; N, 14.95 .

1-(2-Chlorobenzyl)-6-(4-nitrophenyl)pteridine-2,4(1H,3H)-dione

1-(2-Chlorobenzyl)-6-(4-nitrophenyl)lumazine (7d): Yield: 95\%; m.p.: 276-278 ${ }^{\circ} \mathrm{C} ; \mathrm{IR}(\mathrm{KBr}) v_{\max }\left(\mathrm{cm}^{-1}\right)$ : $3179(\mathrm{NH}), 3034$ ( $\mathrm{CH}$ arom), 2912, 2858 ( $\mathrm{CH}$ aliph), 1693 (C=O), 1600 ( $\mathrm{NH}$ bending), 1548, $1397\left(\mathrm{NO}_{2}\right)$, 869 (p.substituted phenyl), 751 (C-Cl); ${ }^{1} \mathrm{H}-\mathrm{NMR}\left(\mathrm{DMSO}-d_{6}\right): \delta 11.99(\mathrm{~s}, 1 \mathrm{H}, \mathrm{NH}), 8.70(\mathrm{~s}, 1 \mathrm{H}, \mathrm{CH}-7)$, 7.50-7.48 (d, 2H, arom), 7.31-7.22 (m, 4H, arom), 6.96-6.94 (d, 2H, arom), 5.09 (s, 2H, $\left.\mathrm{CH}_{2}\right) ;{ }^{13} \mathrm{C}-\mathrm{NMR}$ (DMSO- $d_{6}$ ): $\delta=43.00,107.28,126.08,126.89,127.39,128.23,128.72,129.28,131.41,133.99,140.70,149.10$, 150.38, 150.98, $154.09(\mathrm{C}=\mathrm{O}), 154.75,161.96(\mathrm{C}=\mathrm{O})$; MS: $m / z(\%)=\mathrm{M}+2,411(0.78), \mathrm{M}^{+}, 409$ (1.5), $347(2)$, 303 (3), 256 (24), 150 (79), 72 (33), 44 (100); Anal. calcd. for $\mathrm{C}_{19} \mathrm{H}_{12} \mathrm{ClN}_{5} \mathrm{O}_{4}$ (409.78): C, 55.69; H, 2.95; N, 17.09. Found: C, 55.84; H, 2.94; N, 17.34 .

\subsection{Biological Activity}

\subsubsection{Anticancer Evaluation}

Evaluation of Cytotoxic Effects of the Prepared Compounds

Mammalian cell lines: A-549 cells (human Lung cancer cell line) were obtained from VACSERA Tissue Culture Unit (Cairo, Egypt). Dimethyl sulfoxide (DMSO), crystal violet and trypan blue dye were purchased from Sigma-Aldrich Chemie $\mathrm{GmbH}$ (Taufkirchen, Germany). Fetal Bovine serum, DMEM, HEPES buffer solution, L-glutamine, gentamycin and $0.25 \%$ Trypsin-EDTA were purchased from Lonza (Basel, Switzerland).

Crystal violet stain (1\%) was composed of $0.5 \%(w / v)$ crystal violet and $50 \%$ methanol then made up to volume with $\mathrm{ddH}_{2} \mathrm{O}$.

Cell line Propagation: The cells were propagated in Dulbecco's modified Eagle's medium (DMEM) supplemented with $10 \%$ heat-inactivated fetal bovine serum, $1 \%$ L-glutamine, HEPES buffer and $50 \mu \mathrm{M} / \mathrm{mL}$ gentamycin. All cells were maintained at $37^{\circ} \mathrm{C}$ in a humidified atmosphere with $5 \% \mathrm{CO}_{2}$ and were subcultured two times a week $[40,41]$. 
Cytotoxicity evaluation using viability assay: For cytotoxicity assay, the cells were seeded in a 96-well plate at a cell concentration of $1 \times 10^{4}$ cells per well in $100 \mu \mathrm{L}$ of growth medium. Fresh medium containing different concentrations of the test sample was added after $24 \mathrm{~h}$ of seeding. Different concentrations of the tested chemical compound were added to 96-well, flat-bottomed microtiter plates (Falcon, Somerset, NJ, USA) using a multichannel pipette. The microtiter plates were incubated at $37^{\circ} \mathrm{C}$ in a humidified incubator with $5 \% \mathrm{CO}_{2}$ for of $24 \mathrm{~h}$. Triplicates from each concentration were conducted. Control cells were incubated without sample. After incubation of the cells at $37^{\circ} \mathrm{C}$ for $24 \mathrm{~h}$, the viable cells yield was determined by a colorimetric method [42-44].

Briefly, media were aspirated and the crystal violet solution (1\%) was added to each well for $30 \mathrm{~min}$. The stain was removed, and the plates were rinsed using tap water to remove the excess stain. Glacial acetic acid (30\%) was added to the wells, mixed thoroughly, and the absorbance was measured at $490 \mathrm{~nm}$ using a Microplate reader (SunRise, TECAN, Inc., Mannedorf, Switzerland). The results were normalized to the background absorbance as baseline in wells without stain. Treated samples were compared with the cell control in the absence of the tested compounds. The cell cytotoxic effect of each tested compound was calculated. The viability of cells were determined from the formula $[(\mathrm{ODt} / \mathrm{ODc})] \times 100 \%$ where ODt is the mean optical density of wells treated with the tested sample and ODc is the mean optical density of untreated cells. The relation between surviving cells and drug concentration was plotted to get the survival curve of each tumor cell line for each compound. The $50 \%$ inhibitory concentration $\left(\mathrm{IC}_{50}\right)$ is the concentration of tested compound to stop the growth of $50 \%$ of initial cells. The $\mathrm{IC}_{50}$ values were determined from the dose response curve of each compound with the Graph pad Prism software package (San Diego, CA, USA).

\subsubsection{Molecular Docking Study}

The structures of all tested compounds including the co-crystalized ligands were modeled using the Chemsketch software (Toronto, ON, Canada) (http://www.acdlabs.com/resources/freeware/). The structures were optimized and energy minimized using VEGAZZ software (Milano, Italy), and saved as PDB format. Using AutoDockTools 1.5.6 (La Jolla, CA, USA), all compounds were converted to PDBQT format $[45,46]$. The optimized compounds were used to perform molecular docking against five proteins that represent vital targets for chemotherapeutic drugs, including cyclin dependent kinase-2 (CDK2), B-cell lymphoma 2 (BCL2), Janus kinase 2 (Jak2), p53 binding site in MDM2 (P53) and Dihydrofolate reductase (DHFR). The three-dimensional structure of the molecular target was obtained from Protein Data Bank (PDB) from the website (www.rcsb.org): CDK2 (PDB:1DI8, https: //www.rcsb.org/structure/1DI8), BCL2 (PDB: 2O2F, https://www.rcsb.org/structure/2O2F), Jak2 (PDB: 5AEP, https://www.rcsb.org/structure/5AEP), P53 (PDB: 2LZG, https://www.rcsb.org/structure/2LZG), and DHFR (PDB: 4DFR, https://www.rcsb.org/structure/4DFR). The steps for receptor preparation included the removal of heteroatoms (water and ions), the addition of polar hydrogen, and the assignment of charge. The active sites were defined using grid boxes of appropriate sizes around the bound cocrystal ligands. The docking study was performed using Autodock vina (La Jolla, CA, USA) [47] and Chimera (San Francisco, CA, USA) for visualization [48]. All docking procedures and scoring were recorded according to established protocols [46,49-51].

\section{Conclusions}

In summary, we have developed a new, simple and convenient route for a one pot procedure or even a two or three component reaction. The reaction of 6-amino-1-benzyluracil with benzylidene acetoacetate affords pyridopyrimidine. On the other hand, the reaction of 5,6-diamino-1-2-chlorobenzyl)uracil with different aromatic aldehydes in DMF gave xanthines while the reaction of 5,6-diamino-1-(2-chlorobenzyl)uracil with different aromatic aldehydes and triethyl orthoformate under reflux condition afforded new lumazine derivatives in good yields. The newly synthesized compounds were evaluated for in vivo lung carcinoma inhibitory activity against cell line A549. Compounds 3b, 6c, 6d, 6e, 7c and 7d exhibited the most lung carcinoma inhibitory effect 
compared with the reference drug methotrexate. Molecular-docking analyses revealed that compounds $3 b, 6 c, 6 d, 6 e, 7 c$ and $7 d$ were the best docked ligands against most of the targeted proteins especially CDK2, Jak2, and DHFR proteins, as they displayed the lowest binding energies, critical hydrogen bonds and hydrophobic interactions compared to co-crystalized ligands and methotrexate.

Supplementary Materials: The following are available, Figure S1: Free binding of energy values for the tested compounds docked against CDK2 protein binding site, Figure S2: Free binding of energy values for the tested compounds docked against BCL2 protein binding site, Figure S3: Free binding of energy values for the tested compounds docked against Jak2 protein binding site, Figure S4: Free binding of energy values for the tested compounds docked against-MDM2-P53 protein binding site, Figure S5: Free binding of energy values for the tested compounds docked against DHFR protein binding site, Figure S6: The interaction of the most promising compounds with CDK2 protein, (A) 3D interaction, (B) hydrogen bond formation, and (C) hydrophobic interaction, Figure S7: The interaction of the most promising compounds with Jak2 protein, (A) 3D interaction, (B) hydrogen bond formation, and $(\mathrm{C})$ hydrophobic interaction, Figure S8: The interaction of the most promising compounds with DHFR protein, (A) 3D interaction, (B) hydrogen bond formation, and (C) hydrophobic interaction.

Author Contributions: S.E.-K. formulated the research idea, conceived and prepared the manuscript; S.E.-K. and F.A. performed the experiments and analyzed the data; S.E.-K. wrote the paper. All authors have read and agreed to the published version of the manuscript.

Funding: This work has been funded from our own money.

Acknowledgments: The authors wish to thank Mahmoud Elaasser for carrying out the biological evaluation of this work at the Regional Center for Mycology and Biotechnology at Al-Azhar University, Cairo, Egypt. The authors are also grateful to Mohamed Tantawy for carrying out the molecular modeling (Docking) for the new synthesized compounds at biochemistry division, National Research Centre, El-Buhouth St., Dokki-Cairo, 12622, Egypt.

Conflicts of Interest: The authors declare there is no conflict of interest.

\section{References}

1. Poofery, J.; Khaw-On, P.; Subhawa, S.; Sripanidkulchai, B.; Tantraworasin, A.; Saeteng, S.; Siwachat, S.; Lertprasertsuk, N.; Banjerdpongchai, R. Potential of Thai Herbal Extracts on Lung Cancer Treatment by Inducing Apoptosis and Synergizing Chemotherapy. Molecules 2020, 25, 231. [CrossRef] [PubMed]

2. Barta, J.A.; Powell, C.A.; Wisnivesky, J.P. Global Epidemiology of Lung Cancer. Ann. Glob. Health 2019, 85, 1-16. [CrossRef] [PubMed]

3. Bray, F.; Me, J.F.; Soerjomataram, I.; Siegel, R.L.; Torre, L.A.; Jemal, A. Global cancer statistics 2018: GLOBOCAN estimates of incidence and mortality worldwide for 36 cancers in 185 countries. CA Cancer J. Clin. 2018, 68, 394-424. [CrossRef]

4. Zhou, C.; Zhu, Y.; Lu, B.; Zhao, W.; Zhao, X. Survivin expression modulates the sensitivity of A549 lung cancer cells resistance to vincristine. Oncol. Lett. 2018, 16, 5466-5472. [CrossRef]

5. Jiao, X.-Q.; Qian, X.-L.; Wu, L.; Li, B.; Wang, Y.; Kong, X.; Xiong, L.-X. microRNA: The Impact on Cancer Stemness and Therapeutic Resistance. Cells 2019, 9, 8. [CrossRef]

6. Malhotra, J.; Malvezzi, M.; Negri, E.; La Vecchia, C.; Boffetta, P. Risk factors for lung cancer worldwide. Eur. Respir. J. 2016, 48, 889-902. [CrossRef]

7. Alkhatib, M.H.; Alyamani, S.A.; Abdu, F. Incorporation of methotrexate into coconut oil nanoemulsion potentiates its antiproliferation activity and attenuates its oxidative stress. Drug Deliv. 2020, 27, 422-430. [CrossRef]

8. Alghamdi, H.I.; AlShehri, A.F.; Farhat, G.N. An overview of mortality \& predictors of small-cell and non-small cell lung cancer among Saudi patients. J. Epidemiol. Glob. Health 2017, 7, S1-S6. [CrossRef]

9. Okuyama, A. Lung cancer incidence rates in the world from the Cancer Incidence in Five Continents XI. Jpn. J. Clin. Oncol. 2018, 48, 300-301. [CrossRef]

10. Matsuda, T.; Machii, R. Morphological distribution of lung cancer from Cancer Incidence in Five Continents Vol. X. Jpn. J. Clin. Oncol. 2015, 45, 404. [CrossRef]

11. Zhang, Y.; Qin, Q.; Li, B.; Wang, J.; Zhang, K. Magnetic resonance imaging for N staging in non-small cell lung cancer: A systematic review and meta-analysis. Thorac. Cancer 2015, 6, 123-132. [CrossRef] [PubMed]

12. Chen, M.; Chen, S.; Zhu, F.; Wang, F.; Tian, H.; Fan, Z.; Ke, S.; Hou, Z.; Li, Y. Watson-Crick GC"-inspired supramolecular nanodrug of methotrexate and 5-fluorouracil for tumor microenvironment-activatable self-recognizing synergistic chemotherapy. J. Mater. Chem. B 2020, 8, 3829-3841. [CrossRef] [PubMed] 
13. Ferlay, J.; Soerjomataram, I.; Dikshit, R.; Eser, S.; Mathers, C.; Rebelo, M.; Parkin, D.M.; Forman, D.; Bray, F. Cancer incidence and mortality worldwide: Sources, methods and major patterns in GLOBOCAN 2012. Int. J. Cancer 2015, 136, E359-E386. [CrossRef]

14. Wang, X.; Wang, Y.; Chen, Z.G.; Shin, D.M. Advances of Cancer Therapy by Nanotechnology. Cancer Res. Treat. 2009, 41, 1-11. [CrossRef]

15. Galluzzi, L.; Buqué, A.; Kepp, O.; Zitvogel, L.; Kroemer, G. Immunological Effects of Conventional Chemotherapy and Targeted Anticancer Agents. Cancer Cell 2015, 28, 690-714. [CrossRef]

16. Weissman, I.L. Stem Cells: Biology, Transplantation, and Political Ethics1. Proc. Am. Philos. Soc. 2006, 150, 121-147.

17. Ye, Q.; Liu, K.; Shen, Q.; Li, Q.; Hao, J.; Han, F.; Jiang, R.-W. Reversal of Multidrug Resistance in Cancer by Multi-Functional Flavonoids. Front. Oncol. 2019, 9, 1-16. [CrossRef]

18. Yan, K.-H.; Lee, L.-M.; Hsieh, M.-C.; Yan, M.-D.; Yao, C.-J.; Chang, P.-Y.; Chen, T.-L.; Chang, H.-Y.; Cheng, A.-L.; Lai, G.-M.; et al. Aspirin antagonizes the cytotoxic effect of methotrexate in lung cancer cells. Oncol. Rep. 2013, 30, 1497-1505. [CrossRef] [PubMed]

19. Fotoohi, A.K.; Albertioni, F. Mechanisms of antifolate resistance and methotrexate efficacy in leukemia cells. Leuk. Lymphoma 2008, 49, 410-426. [CrossRef]

20. Duthie, S.J. Folic-acid-mediated inhibition of human colon-cancer cell growth. Nutrition 2001, 17, $736-737$. [CrossRef]

21. Hider, S.L.; A Bruce, I.; Thomson, W. The pharmacogenetics of methotrexate. Rheumatology 2007, 46, 1520-1524. [CrossRef] [PubMed]

22. Hashkes, P.J.; Becker, M.L.; Cabral, D.A.; Laxer, R.; Paller, A.S.; Rabinovich, C.E.; Turner, D.; Zulian, F. Methotrexate: New Uses for an Old Drug. J. Pediatr. 2014, 164, 231-236. [CrossRef]

23. Levêque, D.; Becker, G.; Toussaint, E.; Fornecker, L.-M.; Paillard, C. Clinical pharmacokinetics of methotrexate in oncology. Int. J. Pharmacokinet. 2017, 2, 137-147. [CrossRef]

24. Yoon, S.-A.; Choi, J.R.; Kim, J.-O.; Shin, J.-Y.; Zhang, X.; Kang, J.-H. Influence of reduced folate carrier and dihydrofolate reducatse genes on methotrexate-induced cytotoxicity. Cancer Res. Treat. 2010, 42, 163-171. [CrossRef]

25. Kim, A.; Lee, J.-E.; Jang, W.-S.; Lee, S.-J.; Park, S.; Kang, H.J.; Lee, S.-S. A combination of methotrexate and irradiation promotes cell death in NK/T-cell lymphoma cells via down-regulation of NF- $\mathrm{kB}$ signaling. Leuk. Res. 2012, 36, 350-357. [CrossRef]

26. Pan, Z.; Yang, G.; He, H.; Zhao, G.; Yuan, T.; Li, Y.; Shi, W.; Gao, P.; Dong, L.; Li, Y. Concurrent radiotherapy and intrathecal methotrexate for treating leptomeningeal metastasis from solid tumors with adverse prognostic factors: A prospective and single-arm study. Int. J. Cancer 2016, 139, 1864-1872. [CrossRef] [PubMed]

27. Naidu, M.U.R.; Ramana, G.V.; Rani, P.U.; Mohan, L.K.; Suman, A.; Roy, P. Chemotherapy-Induced and/or Radiation Therapy-Induced Oral Mucositis-Complicating the Treatment of Cancer. Neoplasia 2004, 6, 423-431. [CrossRef] [PubMed]

28. Cronstein, B.N. Low-Dose Methotrexate: A Mainstay in the Treatment of Rheumatoid Arthritis. Pharmacol. Rev. 2005, 57, 163-172. [CrossRef]

29. Tian, H.; Cronstein, B.N. Understanding the mechanisms of action of methotrexate: Implications for the treatment of rheumatoid arthritis. Bull. NYU Hosp. Jt. Dis. 2007, 65, 168-173.

30. Watanabe, K.; Arakawa, Y.; Oguma, E.; Uehara, T.; Yanagi, M.; Oyama, C.; Ikeda, Y.; Sasaki, K.; Isobe, K.; Mori, M.; et al. Characteristics of methotrexate-induced stroke-like neurotoxicity. Int. J. Hematol. 2018, 108, 630-636. [CrossRef]

31. Howard, S.C.; McCormick, J.; Pui, C.; Buddington, R.K.; Harvey, R.D. Preventing and Managing Toxicities of High-Dose Methotrexate. Oncologist 2016, 21, 1471-1482. [CrossRef]

32. Saygin, M.; Ozturk, O.; Ozmen, O.; Ilhan, I.; Gonca, T.; Gumral, N.; Orhan, H.; Aslankoc, R. The impact of methotrexate on lung inflammatory and apoptotic pathway biomarkers-The role of gallic acid. Biomed. Pharmacother. 2016, 84, 1689-1696. [CrossRef] 
33. Guinan, M.; Benckendorff, C.; Smith, M.; Miller, G. Recent Advances in the Chemical Synthesis and Evaluation of Anticancer Nucleoside Analogues. Molecules 2020, 25, 2050. [CrossRef]

34. El-Kalyoubi, S.A.; Agili, F.A. A Novel Synthesis of Fused Uracils: Indenopyrimidopyridazines, Pyrimidopyridazines, and Pyrazolopyrimidines for Antimicrobial and Antitumor Evalution. Molecules 2016, 21, 1714. [CrossRef]

35. El-Kalyoubi, S.; Fayed, E. Synthesis and evaluation of antitumor activities of novel fused tri- and tetracyclic uracil derivatives. J. Chem. Res. 2016, 40,771-777. [CrossRef]

36. El-Kalyoubi, S.A.; Fayed, E.A.; Abdel-Razek, A.S. One pot synthesis, antimicrobial and antioxidant activities of fused uracils: Pyrimidodiazepines, lumazines, triazolouracil and xanthines. Chem. Cent. J. 2017, 11, 1-13. [CrossRef]

37. Youssif, S.; Agili, F. ChemInform Abstract: One-Pot Synthesis of Fused 2-Thiouracils: Pyrimidopyrimidines, Pyridopyrimidines and Imidazolopyrimidines. Z. Nat. B 2008, 63, 860-864. [CrossRef]

38. Marx, D.; Wingen, L.M.; Schnakenburg, G.; Müller, C.E.; Scholz, M.S. Fast, Efficient, and Versatile Synthesis of 6-amino-5-carboxamidouracils as Precursors for 8-Substituted Xanthines. Front. Chem. 2019, 7, 56. [CrossRef]

39. Tantawy, M.A.; El-Sherbeeny, N.A.; Helmi, N.; Alazragi, R.; Salem, N.; Elaidy, S.M. Synthetic antiprotozoal thiazolide drug induced apoptosis in colorectal cancer cells: Implications of IL-6/JAK2/STAT3 and p53/caspases-dependent signaling pathways based on molecular docking and in vitro study. Mol. Cell. Biochem. 2020, 469, 143-157. [CrossRef]

40. Mosmann, T. Rapid colorimetric assay for cellular growth and survival: Application to proliferation and cytotoxicity assays. J. Immunol. Methods 1983, 65, 55-63. [CrossRef]

41. Gomha, S.M.; Riyadh, S.M.; Mahmmoud, E.A.; Elaasser, M.M. Synthesis and Anticancer Activities of Thiazoles, 1,3-Thiazines, and Thiazolidine Using Chitosan-Grafted-Poly(vinylpyridine) as Basic Catalyst. Heterocycles 2015, 91, 1227-1243.

42. El-Sayed, A.S.A.; Yassin, M.A.; Ali, G.S. Transcriptional and Proteomic Profiling of Aspergillus flavipes in Response to Sulfur Starvation. PLoS ONE 2015, 10, e0144304. [CrossRef]

43. Ali, G.S.; Norman, D.; El-Sayed, A.S. Soluble and Volatile Metabolites of Plant Growth-Promoting Rhizobacteria (PGPRs): Role and Practical Applications in Inhibiting Pathogens and Activating Induced Systemic Resistance (ISR). Adv. Bot. Res. 2015, 75, 241-281.

44. El-Sayed, A.S.A.; Ruff, L.E.; Ghany, S.E.A.; Ali, G.S.; Esener, S. Molecular and Spectroscopic Characterization of Aspergillus flavipes and Pseudomonas putida L-Methionine $\gamma$-Lyase in Vitro. Appl. Biochem. Biotechnol. 2017, 181, 1513-1532. [CrossRef]

45. Pedretti, A.; Villa, L.; Vistoli, G. VEGA-An open platform to develop chemo-bio-informatics applications, using plug-in architecture and script programming. J. Comput. Mol. Des. 2004, 18, 167-173. [CrossRef]

46. Kattan, S.W.; Nafie, M.S.; Elmgeed, G.A.; Alelwani, W.; Badar, M.; Tantawy, M.A. Molecular docking, anti-proliferative activity and induction of apoptosis in human liver cancer cells treated with androstane derivatives: Implication of PI3K/AKT/mTOR pathway. J. Steroid Biochem. Mol. Biol. 2020, 198, 105604. [CrossRef]

47. Trott, O.; Olson, A.J. AutoDock Vina: Improving the speed and accuracy of docking with a new scoring function, efficient optimization, and multithreading. J. Comput. Chem. 2009, 31, 455-461. [CrossRef] [PubMed]

48. Pettersen, E.F.; Goddard, T.D.; Huang, C.C.; Couch, G.S.; Greenblatt, D.M.; Meng, E.C.; Ferrin, T.E. UCSF Chimera-a visualization system for exploratory research and analysis. J. Comput. Chem 2004, 25, 1605-1612. [CrossRef]

49. Tantawy, M.A.; Sroor, F.M.; Mohamed, M.F.; El-Naggar, M.E.; Saleh, F.M.; Hassaneen, H.M.; Abdelhamid, I.A. Molecular Docking Study, Cytotoxicity, Cell Cycle Arrest and Apoptotic Induction of Novel Chalcones Incorporating Thiadiazolyl Isoquinoline in Cervical Cancer. Anti-Cancer Agents Med. Chem. 2020, 20, 70-83. [CrossRef] [PubMed]

50. Nafie, M.S.; Tantawy, M.A.; Elmgeed, G.A. Screening of different drug design tools to predict the mode of action of steroidal derivatives as anti-cancer agents. Steroids 2019, 152, 108485. [CrossRef] [PubMed] 
51. El-Far, A.H.; Tantawy, M.A.; Al Jaouni, S.K.; Mousa, S.A. Thymoquinone-chemotherapeutic combinations: New regimen to combat cancer and cancer stem cells. Naunyn-Schmiedeberg's Arch. Pharmacol. 2020, 393, 1581-1598. [CrossRef] [PubMed]

Sample Availability: Samples of the compounds are available from the authors.

Publisher's Note: MDPI stays neutral with regard to jurisdictional claims in published maps and institutional affiliations.

(C) 2020 by the authors. Licensee MDPI, Basel, Switzerland. This article is an open access article distributed under the terms and conditions of the Creative Commons Attribution (CC BY) license (http://creativecommons.org/licenses/by/4.0/). 